\title{
De-regulation of gene expression and alternative splicing affects distinct cellular pathways in the aging hippocampus
}

\author{
Roman M. Stilling ${ }^{1,2+}$, Eva Benito ${ }^{2}$, Michael Gertig $^{2}$, Jonas Barth ${ }^{2}$, Vincenzo Capece ${ }^{3}$, \\ Susanne Burkhardt ${ }^{2}$, Stefan Bonn ${ }^{3}$ and Andre Fischer ${ }^{1,2 *}$ \\ 1 Department of Psychiatry and Psychotherapy, University Medical Center Göttingen, Göttingen, Germany \\ ${ }_{2}^{2}$ Research Group for Epigenetics in Neurodegenerative Diseases, German Center for Neurodegenerative Diseases (DZNE) Göttingen, Göttingen, Germany \\ ${ }^{3}$ Research Group for Computational Analysis of Biological Networks, German Center for Neurodegenerative Diseases (DZNE) Göttingen, Göttingen, Germany
}

Edited by:

Rosanna Parlato, Ulm

University, Germany

Reviewed by:

Hermona Soreq, The Hebrew

University of Jerusalem, Israel

Maria Vittoria Podda, Università

Cattolica del Sacro Cuore, Italy

\section{*Correspondence:}

Andre Fischer, Research Group for

Epigenetics in Neurodegenerative

Diseases, German Center for

Neurodegenerative Diseases

(DZNE) Göttingen, Grisebachstr.

5, 37077 Göttingen, Germany

e-mail: afische2@gwdg.de

${ }^{\boldsymbol{t}}$ Present address:

Roman M. Stilling, Laboratory for

Neurogastroenterology, Alimentary

Pharmabiotic Centre, University

College Cork, Cork, Ireland
Aging is accompanied by gradually increasing impairment of cognitive abilities and constitutes the main risk factor of neurodegenerative conditions like Alzheimer's disease (AD). The underlying mechanisms are however not well understood. Here we analyze the hippocampal transcriptome of young adult mice and two groups of mice at advanced age using RNA sequencing. This approach enabled us to test differential expression of coding and non-coding transcripts, as well as differential splicing and RNA editing. We report a specific age-associated gene expression signature that is associated with major genetic risk factors for late-onset AD (LOAD). This signature is dominated by neuroinflammatory processes, specifically activation of the complement system at the level of increased gene expression, while de-regulation of neuronal plasticity appears to be mediated by compromised RNA splicing.

Keywords: inflammaging, RNA-editing, innate immune system, RNA-seq, neuroinflammation, synaptic plasticity, learning and memory, gene-environment interaction

\section{INTRODUCTION}

Aging is associated with a number of changes that affect cellular homeostasis and impact on the organism's overall health. Aging also leads to a decline of cognitive function including memory formation across species. As such, age-associated memory impairment is observed in invertebrates such as flies as well as in rodents and humans (Horiuchi and Saitoe, 2005; Bishop et al., 2010; Verdaguer et al., 2012). While in humans age is the most significant risk factor for neurodegenerative diseases such as Alzheimer's disease (AD), it is important to note that the degree of cognitive decline varies significantly on an individual level. Thus, some individuals undergo so-called "healthy/successful aging" that is characterized by relatively intact cognitive function, while others develop severe memory impairments and in the most extreme case dementia (Koivisto et al., 1995; Montesanto et al., 2012). In humans it is believed that the manifestation of healthy cognitive aging vs. dementia depends on the variable combinations of genetic pre-disposition and environmental factors an individual experiences throughout lifetime (Fischer, 2014). In order to decipher the molecular signature of age-associated memory impairment it is therefore most suitable to rely on mouse studies in which the genetic background and the environmental factors can be tightly controlled. The mean life span of different mouse strains housed in a laboratory ranges from about 24-30 months (Jucker and Ingram, 1997; Peleg et al., 2010). Previous studies have demonstrated that the onset of age-associated memory impairment in mice can already be observed at 16-18 months of age and is prominent at 24 months of age, while assessment of cognitive function becomes more difficult at more advanced ages due to impaired motor function (Berchtold et al., 2008; Peleg et al., 2010). It has been speculated that age-associated memory decline is correlated to a gene expression signature that dictates cellular plasticity. As such, a number of studies reported altered gene expression in the aging brain using targeted approaches such as qPCR or microarray (Finch and Morgan, 1990; Pletcher et al., 2002; Blalock et al., 2003, 2010; Lu et al., 2004; Verbitsky et al., 2004; Xu et al., 2007; Zahn et al., 2007; Loerch et al., 2008; Pawlowski et al., 2009; Bishop et al., 2010). Unlike these approaches, RNA sequencing is not biased by probe design and in addition to the identification of differential gene expression readily allows the analysis of alternative splicing and RNA editing, two processes intimately linked to cognitive function. RNA sequencing is widely used in other fields and is now also more commonly applied to study brain tissue (Dillman et al., 2013; Mazin et al., 2013; Wood et al., 2013; Stilling et al., 2014). However, the aging hippocampus, a key region for memory formation in rodents and humans that is affected early in ageassociated memory decline and $\mathrm{AD}$, has not been studied using RNA sequencing. To this end, we used Illumina next-generation sequencing to compare the hippocampal transcriptomes of 3,24 , and 29-month-old C57BL/6J mice (3M, 24M, and 29M, respectively). We find that the aging hippocampus is characterized by 
a strong neuroinflammatory gene-expression signature that is dominated by differential gene expression but not by differential splicing or RNA-editing. A key component of the neuroinflammatory response was activation of the complement system that has repeatedly been genetically linked to AD (Bertram et al., 2007; Lambert et al., 2009; Brouwers et al., 2012). Taking into account that neuroinflammation is a key mechanism in neurodegenerative diseases, our data supports the view that $\mathrm{AD}$ may represent accelerated brain aging due to an unfavorable genetic pre-disposition and exposure to environmental risk factors. On the other hand, we find that compromised synaptic function is linked predominantly to alternative splicing suggesting that, on the level of the transcriptome, age-associated neuroinflammation and decreased synaptic plasticity are mediated by distinct cellular processes.

\section{MATERIALS AND METHODS ANIMALS}

Specific pathogen free (SPF) C57Bl6/J wild type mice were obtained from Janvier SAS. Mice were kept in groups $\leq 5$ animals in individually ventilated cages $(32 \times 16 \times 14 \mathrm{~cm}$, Techniplast $)$ on a $12 \mathrm{~h}$ light/dark cycle with food and water ad libitum. To obtain mice between 28 and 30 months of age, 24 month-old mice were ordered from Janvier SAS and kept in our holding rooms for 4-6 months. All procedures were performed by experienced experimenters and according to protocols approved by the Lower Saxony State Office for Consumer Protection and Food Safety.

\section{NOVEL OBJECT RECOGNITION}

Behavioral testing was performed as described previously (Kerimoglu et al., 2013). Animals were habituated individually to a uniform-gray plastic arena $(90 \times 90 \mathrm{~cm}$, walls $20 \mathrm{~cm}$ high $)$ for $5 \mathrm{~min}$ on two subsequent days. Animals were then further habituated to two equal objects placed in opposing corners of the arena for $5 \mathrm{~min}$ on the next two days. On day 5 objects were exchanged by two new but equal objects $(\mathrm{A}+\mathrm{A})$ and animals were allowed to explore the objects for $5 \mathrm{~min}$. Then, mice were sent back to their home cages for $5 \mathrm{~min}$ (for short-term memory assessment) and reintroduced to the arena after one object was exchanged (objects A + B). After $24 \mathrm{~h}$, object B was exchanged for object $C$ for long-term memory assessment. Duration of object contacts was measured. Mice that only showed summed contact time of $<1 \mathrm{~s}$ were excluded from the analysis of this test. Object preference was defined as (novel object)/sum(both objects).

\section{RNA EXTRACTION AND SEOUENCING}

Total RNA was extracted using TRI Reagent (Sigma-Aldrich) as previously described (Peleg et al., 2010). In brief, flash-frozen tissue was homogenized on ice with several pestle strokes in $0.5 \mathrm{ml}$ of TRI Reagent (Sigma-Aldrich) After addition of another $0.5 \mathrm{ml}$ of TRI Reagent and $5 \mathrm{~min}$ incubation at room temperature (RT) the dissociated homogenate was mixed with $300 \mu \mathrm{l}$ of $\mathrm{CHCl} 3$ and incubated for $15 \mathrm{~min}$ (RT) followed by centrifugation at $12,000 \times \mathrm{g}\left(4^{\circ} \mathrm{C}\right)$. The upper aqueous phase was transferred to a new tube, mixed with $500 \mu \mathrm{l}$ isopropanol and incubated at $-20^{\circ} \mathrm{C}$ for at least $1 \mathrm{~h}$ for precipitation. RNA was precipitated by centrifugation at $12,000 \mathrm{~g}$ for $30 \mathrm{~min}\left(4^{\circ} \mathrm{C}\right)$. The pellet was washed twice with $1 \mathrm{ml}$ of $75 \%$ ethanol (centrifugation after washing steps: $12,000 \times \mathrm{g}, 5 \mathrm{~min}, 4^{\circ} \mathrm{C}$ ). The washed pellet was dissolved in $30 \mu \mathrm{l}$ of RNase-free water. Following DNase1 (life Technologies) treatment to remove residual contaminating genomic DNA for $20 \mathrm{~min}$ at $37^{\circ} \mathrm{C}$, RNA was purified using phenol-chloroform extraction. Library preparation and cluster generation for mRNA sequencing [single-end libraries for $3 \mathrm{M}$ $(n=5)$ vs. $24 \mathrm{M}(n=6)$ comparison; paired-end libraries for $3 \mathrm{M}$ vs. $29 \mathrm{M}$ comparison $(n=3)]$ was performed according to Illumina standard protocols using the TruSeq RNA Sample Prep Kit v2 and the TruSeq Paired-End Cluster Generation Kit v3-cBot-HS (for paired-end mRNA-seq) with subsequent use of the corresponding TruSeq Cluster Generation Kit v3cBot-HS (for single-end mRNA-seq). Libraries were quality controlled and quantified using a Nanodrop 2000 (Thermo Scientific), an Agilent 2100 Bioanalyzer (Agilent Technologies) and Qubit (life Technologies). For the sequencing run, TrueSeq SBS kits were used according to Illumina manuals. Read lengths were $1 \times 50$ bp for single-end and $2 \times 100$ bp for paired-end sequencing.

\section{BIOINFORMATIC ANALYSIS PIPELINE Differential gene expression}

Differential gene expression analysis of RNA sequencing data was performed as described previously (Stilling et al., 2014). In brief, library preparation [single-end libraries for 3-month $(3 \mathrm{M}) \quad(n=5) \quad$ vs. 24 -month $(24 \mathrm{M}) \quad(n=6)$ comparison; paired-end libraries for $3 \mathrm{M}$ vs. 29-month (29M) comparison $(n=3)$ ] and cluster generation for mRNA sequencing were performed as required by Illumina protocols (TruSeq, Illumina). Downstream analysis steps after read retrieval included quality control (FastQC, www.bioinformatics.babraham.ac.uk/projects/ fastqc/) and mapping to reference genome (STAR aligner v2.3.0, Dobin et al., 2013). For calling of differentially expressed genes (DEG), mapped reads were counted with HTSeq v0.5.4p2 (http://www-huber.embl.de/users/anders/HTSeq) (non-default parameters: -m intersection non-empty) and count tables were analyzed independently for both aging groups vs. the $3 \mathrm{M}$ young control group using the DESeq2 v1.2.5 R-package (Anders and Huber, 2010). Genes with a $\log _{2}$ (fold-change) $\geq 0.5$ and adjusted $p$-value $\leq 0.05$ were considered differentially regulated. All expression data are made publicly available in a GEO SuperSeries (GSE61918). Compressed fastq-files and primary analysis can be found under GEO accession GSE61915, microarray raw data is available under GEO accession GSE61647.

\section{Functional annotation}

Functional annotation and category and pathway analysis was carried out using the Database for Annotation, Visualization, and Integrated Discovery (DAVID, v6.7) (Huang et al., 2009a,b). Noncutoff-based gene set enrichment analysis (GSEA) was performed by using the Broad Institute GSEA application (Mootha et al., 2003; Subramanian et al., 2005). Input lists for GSEA were ranked based on $\log _{2}$-fold-change.

\section{Transcription factor binding sites}

Promoter analysis to search for overrepresented transcription factor binding sites (TFBS) was done using the Pscan web interface 
(Zambelli et al., 2009) (http://159.149.160.51/pscan/) scanning the promoter region from $-250 \mathrm{bp}$ to $+50 \mathrm{bp}$ from TSS comparing to JASPAR descriptors. All Gene ID conversion was done using BioMart database queries (www.ensembl.org/biomart/).

\section{Differential exon usage}

For detection of differential exon usage the DEXSeq R-package was used (Anders et al., 2012) with default parameters. FDRcorrected $p$-value significance level was set to 0.01 for the $3 \mathrm{M}$ vs. $24 \mathrm{M}$ comparison and to 0.1 for the $3 \mathrm{M}$ vs. $29 \mathrm{M}$ comparison to adjust for differences in library-type and sample size.

\section{RNA-editing}

Known RNA-editing positions in the mouse reference genome (mm10/GRCm38 coordinates) were retrieved from previously described editing sites in the mouse genome from two online databases and two recent publications and compiled to a nonredundant list of 17831 positions (Table S6). This list was used as input for the REDItools algorithm REDItoolKnown.py (Picardi and Pesole, 2013). Further input arguments were a list of splice sites (taken from UCSC table browser), the current genome sequence (GRCm38p2) in fasta format and the most recent GTF file (taken from Ensembl, version GRCm38.e75). Non-default parameters were $-\mathrm{C} 1000,-\mathrm{c} 0,-\mathrm{q} 10,-\mathrm{m} \mathrm{10},-\mathrm{v}$ $1,-\mathrm{n} 0.001,-\mathrm{t} 4$. For statistical comparison between groups independent, two-sided Student's $t$-tests were used for each position.

\section{MICROARRAY}

RNA quality control, cDNA synthesis, mono-color Cy3-labeling and hybridization to whole mouse genome microarray chips were carried out according to Standard Operating Procedures of the Transcriptome Core facility at University of Göttingen. Total RNA was labeled with Cy3 according to Agilent's Low RNA Input Fluorescent Linear Amplification Kit and later hybridized to Agilent Whole Mouse Genome $4 \times 44 \mathrm{~K}$ G4122F microarrays according to the manufacturer's protocol. Quantity and Cy3dye incorporation rates of the generated target material were assessed using a NanoDrop ND-1000. Washes were performed according to the Agilent Technologies SSPE protocol (v2.1) wash solution 3 was replaced by acetonitrile. After that, scanning was performed using an Agilent G2505B scanner. Data analysis was performed as described previously (Peleg et al., 2010; Agis-Balboa et al., 2011; Kerimoglu et al., 2013). In summary, data was analyzed using Agilent Feature Extraction software, version 9.5.3.1 and the Limma (Smyth et al., 2005) package for $\mathrm{R} /$ Bioconductor (Gentleman et al., 2004). In order to assure that the intensities had similar distributions across arrays, VSN normalization (Huber et al., 2002) was applied to the intensity values as a method for between-array normalization. To estimate the average group values for each gene and assess differential gene expression, a simple linear model was fit to the data, and group-value averages and standard deviations for each gene were obtained. To find genes with significant expression changes between groups, empirical Bayes statistics were applied to the data by moderating the standard errors of the estimated values (Smyth, 2004). $P$-values were inferred from the moderated $\mathrm{t}$-statistic and corrected for multiple testing using the FDR method (Benjamini and Hochberg, 1995). Afterwards, the final output was filtered for probes showing a change in normalized intensity that was greater than $1.414-$ fold $\left[\log _{2}\right.$ (fold change $\left.) \geq 0.5\right]$ with an adjusted $p$-value of $\operatorname{FDR}(\mathrm{p})<0.1$.

\section{QUANTITATIVE REAL-TIME PCR (qRT-PCR)}

Quantitative real-time PCR ( $\mathrm{qPCR}$ ) was performed as described before (Peleg et al., 2010). In summary, $1 \mu \mathrm{g}$ of total RNA was used for CDNA synthesis and CDNA was diluted 1:10. Probe-based qRT-PCR (UPL, Roche) was carried out on a LightCycler 480 II (Roche) and analyzed using suppliers software. Primers used for $C 4 b$ amplification: Fwd(5'-TCTCACAAACCCCTCGACAT-3'), $\operatorname{Rev}\left(5^{\prime}\right.$ - AGCATCCTGGAACACCTGAA-3'), UPL-Probe \#10.

\section{IMMUNOHISTOCHEMISTRY}

Fluorescent staining of target proteins was performed as previously described (Peleg et al., 2010). In brief, mice were transcardially perfused with 4\% PFA, brains isolated and postfixed for another $16 \mathrm{~h}$ in $4 \%$ PFA. Free-floating cryosections $(30 \mu \mathrm{m})$ were incubated with $5 \%$ goat serum for blocking and followed by incubation with target-specific primary antibodies (anti-NeuN [A60, MAB377, Merck Millipore, 1:1000, antiGFAP [G5601, Promega, 1:1000], anti-IBA1 [019-19741, WAKO, $1: 1000])$. Corresponding secondary antibodies were from life Technologies (anti-mouse Alexa-488 labeled, A11029; anti-rabbit Alexa-633 labeled, A21071). Images were taken on a Leica SP2 confocal microscope. Stereological analysis of the number of cells was performed on 4 serial $40 \mu \mathrm{m}$ free-floating coronal sections per animal which were analyzed by confocal microscopy to count cells expressing the indicated marker. Cell number was assessed as areal density across the CA1 region. The data was normalized to the 3 month groups.

\section{RESULTS \\ DIFFERENTIAL GENE EXPRESSION ANALYSIS IN THE AGING HIPPOCAMPUS}

Age-associated memory impairment is the result of variable combinations of genetic pre-disposition and environmental factors, which eventually causes detrimental changes in cellular homeostasis. We therefore reasoned that a comprehensive picture of age-related changes in transcription would be most informative about the aging processes occurring in the brain. The hippocampal formation is essential for memory function in rodents and humans and has been linked to cognitive age-associated memory impairment (Fanselow, 2010). Thus, we performed deep sequencing of polyA-enriched RNA extracted from the mouse hippocampus in three different age groups (3-month-old mice, 24-month-old-mice, and 29-month-old-mice). Since C57BL/6J mice in the laboratory have a maximum life span of just above 30 months, 24-month-old mice represent a model of advanced aging, while 29-month-old represent a time point at the end of life-span. We first confirmed that the selected groups of mice indeed show age-associated memory decline (Figure 1). Due to severely impaired locomotor activity in 29-month-old mice we had to exclude these mice from any behavioral testing. A commonly employed test for hippocampus-dependent memory 


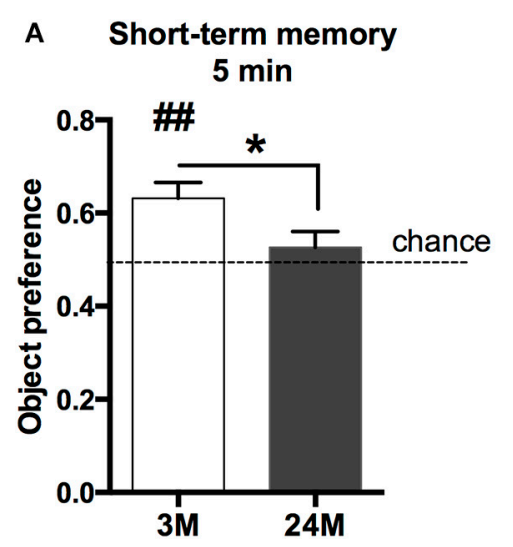

B Long-term memory $24 \mathrm{hrs}$

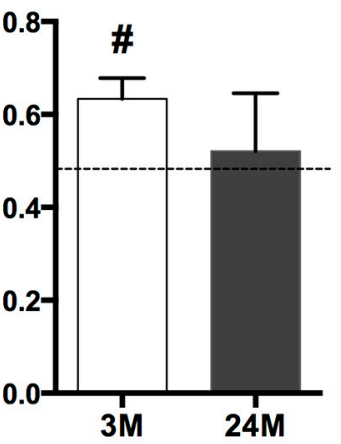

C Training

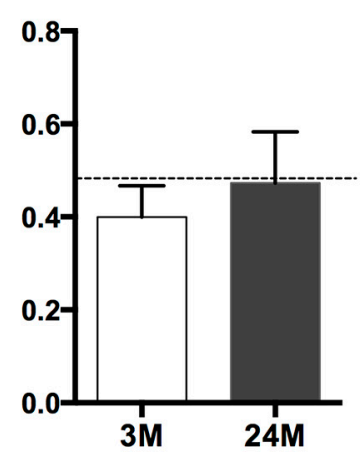

FIGURE 1 | Impaired novel object recognition memory in 24-month-old mice. (A) 3-month-old mice (3M) and 24-month-old mice (24M) were tested in the novel object recognition paradigm. To assay short-term memory, mice were first exposed to two similar objects $(A+A)$ and then re-exposed to the testing area containing one novel object 5 min later $(A+B)$. Preference for the novel object was significantly greater in 3-month-old mice when compared to the 24-month group, which performed at chance level $(* p<0.05$ between groups, two-sided t-test; $\#$ \# 0.01 vs. chance level, one-sample $t$-test; $n=11[3 \mathrm{M}] / 9[24 \mathrm{M}]$ ). (B) To test long-term memory mice were exposed to the arena $24 \mathrm{~h}$ later, now containing another novel object $(A+C)$. While object preference in $3 \mathrm{M}$ was significantly higher than chance level $(\# p<0.05$, one-sample $t$-test, $n=10), 24 \mathrm{M}$ animals $(n=4)$ performed at chance level, indicating impaired long-term object recognition memory in aged mice. Error bars indicate s.e.m. (C) During the training session, none of the groups showed preference for any of the two equal objects $(A+A)$. function in mice is the Morris water maze test. Pilot experiments however showed that in our hands even 24-month-old mice have difficulties to cope with the 2-week-lasting daily training procedure, in which the animals have to swim in a pool filled with opaque water and need to find and climb on a hidden platform. Thus, we decided to subject mice to the novelobject-recognition paradigm that does not depend on advanced motor skills and allows the measurement of short and longterm memory in a non-stressful experimental setting. Moreover, while object recognition learning recruits various brain structures, it also depends on an intact hippocampus (Broadbent et al., 2010; Antunes and Biala, 2012). As expected, we observed that both short (Figure 1A) and long-term object-recognition memory (Figure 1B) was impaired in 24-month-old mice, when compared to 3-month-old mice. This was not due to altered explorative behavior during the training, since both groups of mice explored the objects presented during the training session to a similar degree (Figure 1C).

In the next step, we isolated total RNA from the hippocampi of 3-, 24-, and 29-month-old mice and subjected it to RNA sequencing. In line with previous studies, we observed that aging was not associated with massive changes in cell number (Long et al., 1999) (Figure S1). We first compared differential gene expression across the different age groups using the 3 month group as reference. 477 genes were differentially expressed (313 up-regulated, 164 down-regulated) in 24-month-old mice and 323 genes (275 up-regulated, 48 down-regulated) in 29month-old mice (Figure 2A, Table S1) when compared to the 3-month group. In all comparisons, we observed a general trend toward higher numbers of up-regulated genes $(\sim 70 \%)$ compared to down-regulated genes (Figure 2B). When we compared these lists among each other, we found a significant amount of overlap between genes up-regulated at 24 and 29 months of age (122 genes, Figure 2C, Table S1). The overlap between the genes down-regulated in 24- and 29-month-old mice was less pronounced (17 genes, Figure $2 \mathrm{C}$, Table S1). When we analyzed the 122 genes commonly up-regulated in 24- and 29-month-old mice for functional pathways we observed inflammatory signaling pathways, namely the "Systemic lupus erythematosus" and the "complement and coagulation pathway" to be highly enriched (Figure 2D, Table S2). It has to be noted that several genes of the complement system are linked to systemic lupus erythematosus, which explains the enrichment of this pathway (Table S2). This data suggests that activation of the complement system is one of the key features of the aging hippocampus. Similar results were obtained when we separately analyzed all genes upregulated in 3 vs. 24-month-old mice (Figure 2E, left panel). When we analyzed all up-regulated genes in 3 vs. 29-month-old mice, we found significant enrichment of additional immunerelated pathways (Figure 2E, right panel, blue bars), suggestive of an even more pronounced immune activation with increasing age. Another group of up-regulated genes was associated with cell adhesion since "cell adhesion molecules" was also identified as a significantly enriched pathway in 24- and 29-month-old mice (Figure 2E, Table S2).

These findings, based on analysis of lists of significantly up-regulated genes using the DAVID platform, were further confirmed by GSEA, an unbiased approach to analyze enrichment of functional groups in a given gene list without the need for thresholding or introduction of $p$-value cutoffs. Similar to the previous analysis, we found the enriched immune system pathways, including the top-enriched "complement and coagulation pathway" in both aging groups (Figure $2 \mathrm{~F}$ ), as well as several additional pathways associated with immunesystem function (Figure S2). Interestingly, several of these additional categories were associated with pathological infection by bacteria, viruses and other pathogenic organisms, suggesting that at least part of the neuroinflammatory response may 
A

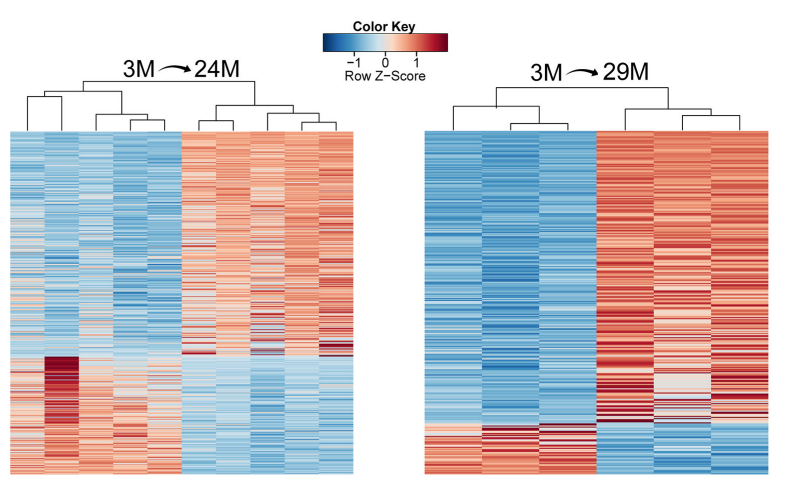

C

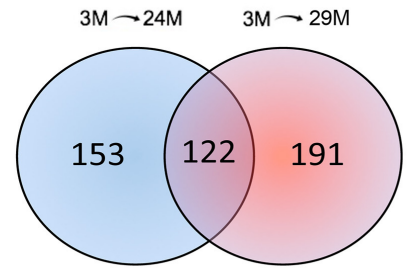

up-regulated genes

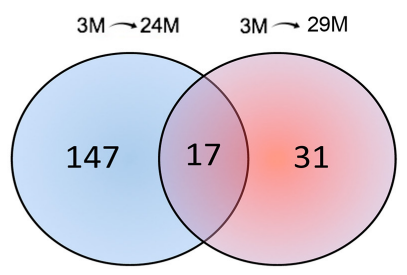

down-regulated
B

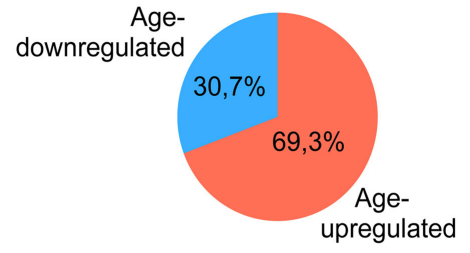

D

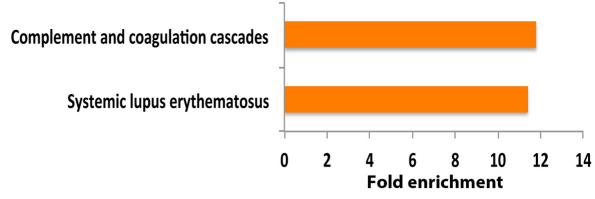

Upregulated KEGG Pathways 29M

Upregulated KEGG Pathways 24M

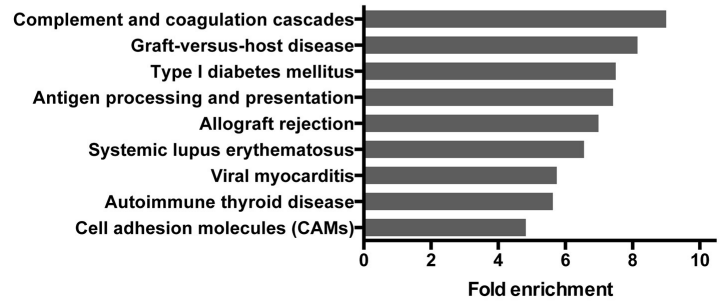

$\mathbf{F}$

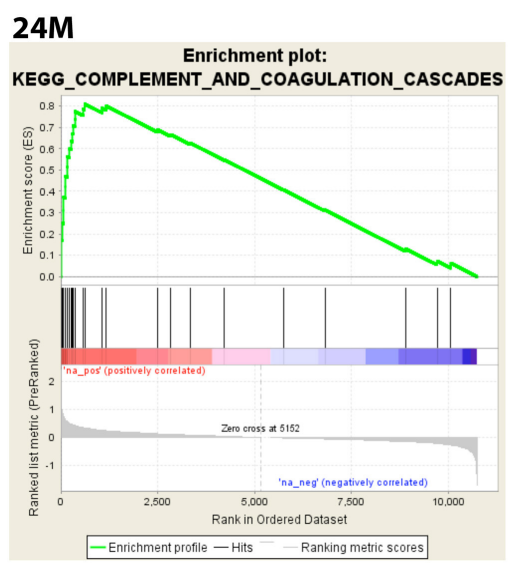

$29 \mathrm{M}$

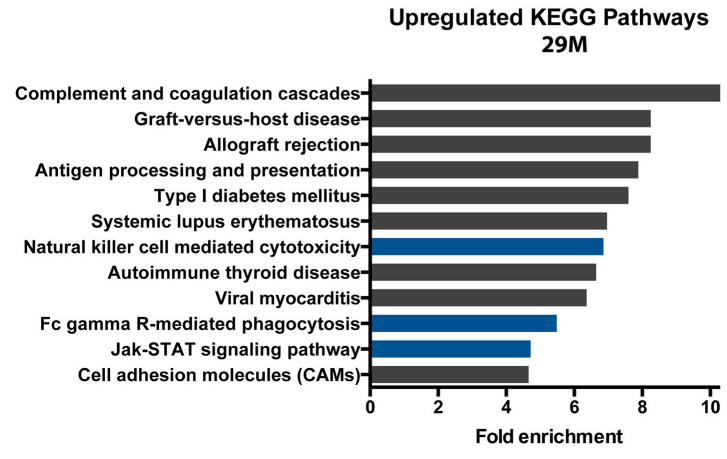

G
KEGG_COMPLEMENT_AND_COAGULATION_CASCADES

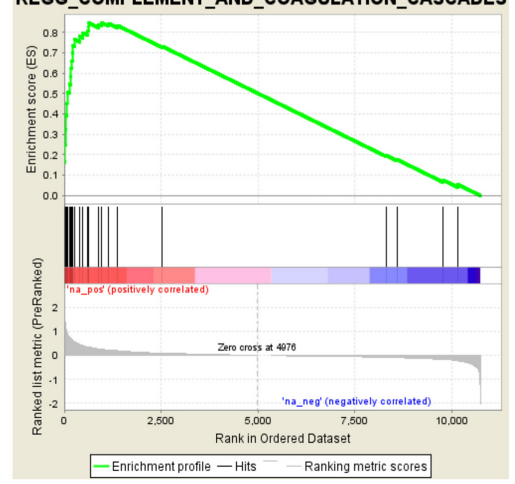

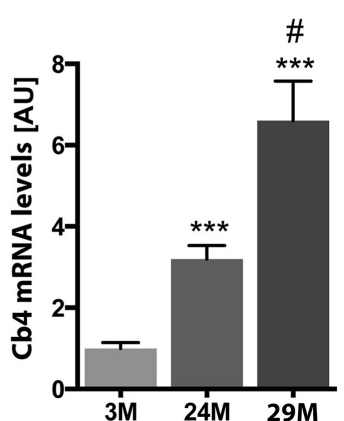

FIGURE 2 | Aging is associated with changes in gene expression and upregulation of immune system functions in the hippocampus.

(A) Heatmaps of differentially expressed genes in 3-month (3M) and

24-month-old mice (24M, left panel) and 3 and 29-month (29M) old mice (right panel). High expression is marked by the red color spectrum, low expression by blue colors. (B) Pie chart showing the percentage of genes up-and down-regulated in 3 vs. 24/29 month-old mice. (C) Venn diagrams comparing significantly regulated genes found in the different experiments (3M vs. $24 \mathrm{M}$ and 3M vs. 29M). (D) Genes up-regulated at 24 and 29 month of age were subjected to functional enrichment analysis for overrepresented biological processes and KEGG pathways. (E) Fold enrichment of overrepresented KEGG pathways in up-regulated genes when individually comparing the $3 \mathrm{M}$ vs. $24 \mathrm{M}$ and $3 \mathrm{M}$ vs. $29 \mathrm{M}$ groups. (F) Using non-cutoff gene set enrichment analysis (GSEA), the top up-regulated KEGG pathway was the complement and coagulation cascade. Shown are enrichment plots along expressed genes,

(Continued) 
FIGURE 2 | Continued

ranked by fold-change [left/red: positive $\log _{2}$ fold-change (upregulation), right/blue: negative $\log _{2}$-fold-change (downregulation); a gene contributing to the enrichment score of the selected pathway along the ranked list is marked by a vertical black line and increases the cumulative enrichment score (green line)]. (G) QPCR analysis of the $C 4 b$ gene ${ }^{* * * *} p<0.001$ vs. $3 \mathrm{M}$ group, two-sided $t$-tests; ${ }^{\#} p<0.05$ vs. $24 \mathrm{M}$ group, two-sided $t$-test; $n=$ $5[3 \mathrm{M}] / 4[24 \mathrm{M}] / 8[29 \mathrm{M}])$. Error bars indicate s.e.m. be attributed to the invasion of parasitic microbes across the blood brain barrier. Furthermore, GSEA found a similar set of additional pathways in the 29-month-old mice compared to the 24-month group as detected by DAVID platform analysis. Thus, GSEA confirmed functional enrichment of immune system-related pathways, which are gradually up-regulated with advancing age.

To further verify these findings we decided to reproduce the results in an additional cohort of 3 and 29-month-old mice using a microarray approach thereby also controlling for any potential experimental bias that might be introduced by RNA sequencing. Albeit DEG differed to some extend, the affected pathways were almost identical and the complement system was the most affected pathway among the up-regulated genes (Table S2).

Within this pathway the $C 4 b$ gene encoding the complement factor 4 (C4) constantly turned up among the most significantly up-regulated genes in all our analyses. Thus, to validate the finding made by genome-wide techniques we sought to directly compare expression levels of this gene among all three aging groups by qRT-PCR. We detected a 3.2-fold increase of $C 4 b$ mRNA levels in the 24-month $(24 \mathrm{M})$ group and a further increase in 29 -month (29M) group (6.6-fold) when compared to the 3-month (3M) group (Figure 2G).

We also determined functional enrichment among downregulated genes. Interestingly, the down-regulated genes in the $24 \mathrm{M}$ group were enriched for genes of the "neuroactive ligandreceptor interaction" and "regulation of transcription" pathways, while no pathways could be detected in the $29 \mathrm{M}$ group, even if we combined the data obtained by RNA sequencing and microarray (Table S2). When we combined the genes down-regulated in 24- and 29-month-old mice we observed the "neuroactive ligandreceptor interaction" and the "calcium signaling" pathways to be significantly affected (Table S2).

In addition to protein-coding genes, we could also detect differential regulation of several non-coding RNAs (ncRNAs), including the pseudogene Pisd-ps1 and the long intergenic noncoding RNA (lincRNA) Neat1 as well as Malat1, also known as Neat2 (Table S1). While the role of long non-coding RNAs is only beginning to emerge, Neat 1 and Malat 1 are known to accumulate in the nucleus, where they form the RNA backbone of so-called paraspeckles, subnuclear ribonucleoprotein bodies involved in transcriptional regulation, e.g., by nuclear retention of RNAs (Bond and Fox, 2009). Interestingly, we could also detect increased transcription from the $C 4 a$ locus in the $29 \mathrm{M}$ group. $C 4 a$ is closely related to the nearby $C 4 b$ but does not encode a protein, according to the ENSEMBL database. Though their functional significance is subject to ongoing research, these results indicate that also regulatory ncRNAs are implicated in hippocampal aging.

Taken together, analysis of differential gene expression revealed that hippocampal aging is markedly characterized by up-regulation of a neuroinflammatory program. Especially activation of the complement system was highly correlated with increasing age.

\section{AGE-ASSOCIATED CHANGES IN GENE EXPRESSION ARE ORCHESTRATED BY A SPECIFIC SET OF TRANSCRIPTIONS FACTORS}

To further elucidate potential upstream mechanisms of the identified transcriptional program, we searched the promoters of regulated genes for common TFBS. We found a large number of potential TFBS significantly enriched at promoters of genes that were up-regulated with aging (Table S3). Of these, the TOP20 most strongly enriched TFBS were largely similar in the $24 \mathrm{M}$ and $29 \mathrm{M}$ group (Figures $3 \mathbf{A}, \mathbf{B}$ ). We could identify a number of common transcription factor families that together made up the bulk of the significantly enriched TFBS [Signal transducer and activator of transcription (STATs), Interferon regulatory factor (IRF), Spleen focus forming virus proviral integration oncogene (SPI), Activator protein 1, (AP1, composed of Fos, Jun and ATF family members), Nuclear factor kappa-light-chain-enhancer of activated B cells (NF$\kappa \mathrm{B}$ ), the GATA and the E26 transformation-specific (ETS) family of transcription factors (Table S3)], which are strongly associated with their roles in immune-related signaling (Peng, 2008). Of note, this set of TFs explained $44 \%$ of all up-regulated genes in 24 - and $53 \%$ in 29 -month-old mice. In fact, up to $87 \%$ of all upregulated genes could be assigned to the action of enriched TFs (Figure 3C, Table S3).

In agreement with functional enrichment of DEG, we observed a higher diversity of enriched TFBS in the 29M group (Figure 3B, Table S3). While TFBS of the AP1 family were more abundant in the $24 \mathrm{M}$ group, TFBS of the NF- $\kappa \mathrm{B}$ and GATA families were more abundant in the 29-month group. Notably, also the Z-scores and significance levels for the STAT and IRF families were considerably higher in the $29 \mathrm{M}$ group (Figures $3 \mathrm{~A}, \mathbf{B}$ Table S3), which was in line with up-regulation of the Stat1 gene and several Irf genes in the 29M group (Table S1). Together, these results suggest increasing usage of STAT- and IRF-related cellular signaling pathways in the hippocampus with increasing age.

Among down-regulated genes, significant enrichment of TFBS was less pronounced and more heterogeneous. However, binding sites for the E2F family of transcription factors as well as for the specificity protein (SP) and EGR families were identified as common between the two aging groups and enrichment of EGR TFBS among down-regulated genes correlated with down-regulation of Egr genes in the 24-month group (Tables S1, S3).

\section{WIDESPREAD AND SPECIFIC ALTERNATIVE EXON USAGE CHANGES IN THE AGING HIPPOCAMPUS}

Alternative to the altered expression of genes, co-transcriptional intron excision from the nascent pre-mRNA, known as splicing, is the main mechanism for generation of transcript isoforms and differential exon use in information-dense and complex genomes, 


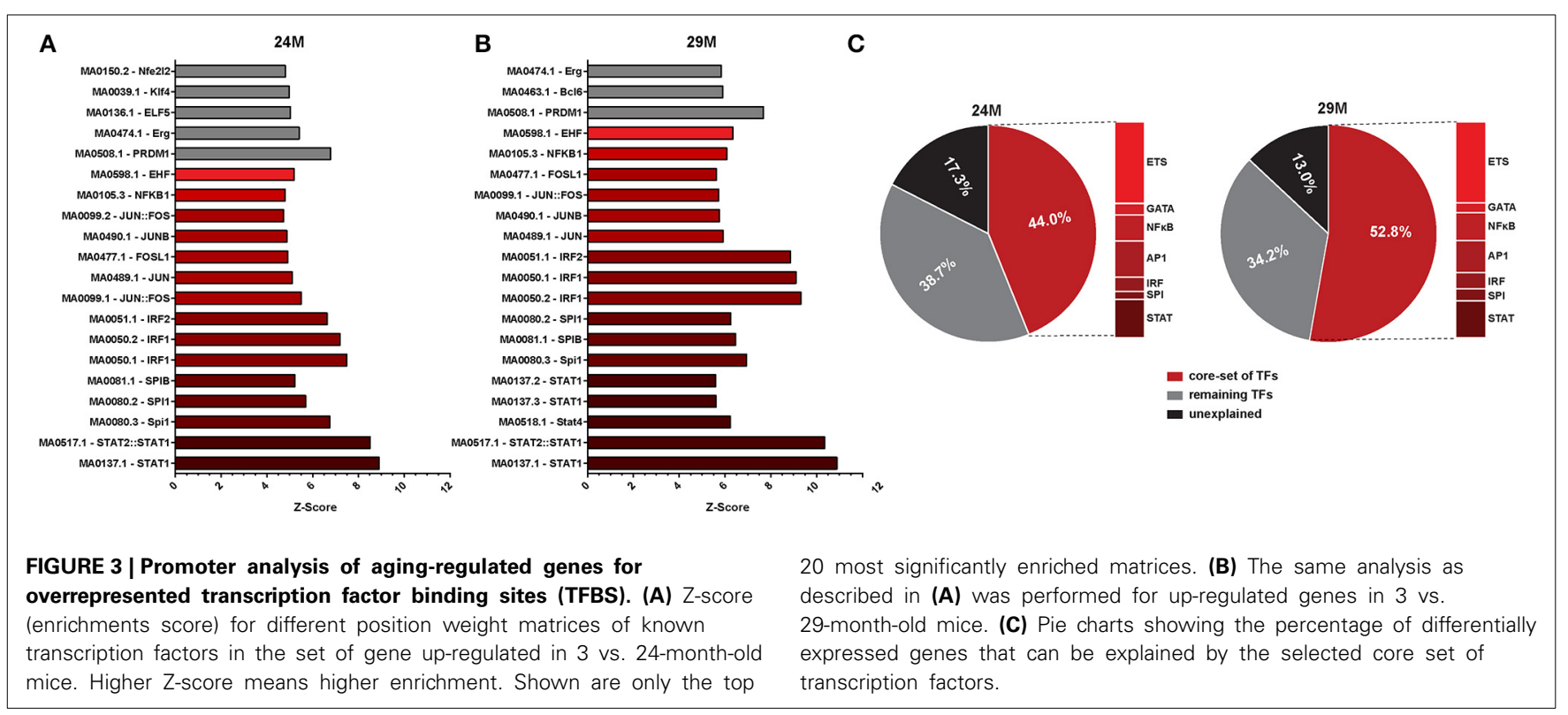

thereby adding another important level of gene expression control.

We therefore analyzed our RNA sequencing data with respect to differential exon usage. We found 436 annotated genes with significant changes in exon usage in the $24 \mathrm{M}$ group and 80 genes in the 29M group (Figure 4A; Table S4). Thus, changes in RNA splicing were, at least in the $24 \mathrm{M}$ group, quantitatively comparable to the changes observed in gene expression (Figure 4A). However, there was little to no overlap between genes affected by altered splicing and genes that were differentially expressed (Figure 4B). In fact, less than $1 \%$ of the DEG were also differently spliced in the $24 \mathrm{M}$ group, while zero overlap was found in the $29 \mathrm{M}$ group. This data indicates that differential gene expression and alternative splicing may affect different signaling pathways. To test this hypothesis, we analyzed the differentially spliced genes for enrichment of functional pathways. Our analysis revealed that there was a significant overrepresentation of genes associated with neuronal function including synaptogenesis, regulation of synaptic transmission, axonogenesis, neuron projection morphogenesis, postsynaptic density and long-term potentiation (Figure 4C, Table S5). Of note, none of these pathways was enriched in gene-set of DEG (Figure 4C). Vice versa, pathways linked to inflammatory response-which dominated the list of DEG-could not be identified within the group of alternatively spliced genes (Figure 4C). These data suggest that inflammatory responses in the aging hippocampus are driven by changes in differential gene expression whereas de-regulation of synaptic plasticity in mainly attributed to differential splicing. One of the genes that was differentially spliced in both 24- and 29-monthold mice when compared to their young counterparts was the Spectrin $\beta$, non-erythrocytic 1 gene (Sptbn1), that showed a specific upregulation in usage of exon 10 with age (Figure 4D). Sptbn1 is best known for its role in cytoskeleton regulation during neurite outgrowth (Lee et al., 2012), which is in agreement with our previous functional enrichment analysis. The inclusion of exon 10 suggests a shift towards higher expression of $S p t b n 1$ isoform 2, resulting in a protein that has shorter and distinct $\mathrm{N}$ - and $\mathrm{C}$ - termini compared to isoform 1 and lacks the pleckstrin homology (PH) domain that is critical for tethering F-actin filaments to the plasma membrane (Figure S3).

In addition to these widespread changes, we found differential exon usage in the amyloid beta (A4) precursor protein (App) and the beta-site APP cleaving enzyme 1 (Bace1) genes (Table S4), both of which are strongly implicated in the etiology of AD. This suggests that also differential-splicing events that occur with aging may be implicated with altered $\mathrm{A} \beta$ production in $\mathrm{AD}$.

\section{DIFFERENCES IN RNA-EDITING}

A second mechanism to generate alternative transcript isoforms is RNA-editing. Though more subtly changing the coded information as compared to alternative splicing, it also occurs co-transcriptionally and may lead to altered regulation of the transcript or a change in amino acid sequence of the encoded protein. Notably, the mammalian, and especially the human, brain has been identified as the principal site for RNA-editing. Since RNA sequencing is not only able to quantify transcript abundance but also allows visualization of the exact sequence of these transcripts, it is possible to identify changes in transcript sequence from the genomic reference at known positions. We compiled a list of previously described editing sites in the mouse genome from two online databases and two recent publications to yield a non-redundant list of 17831 positions (Table S6).

At 682 of the 17831 known positions, we found RNA-editing in the $3 \mathrm{M}$ group (Table S7). Of these 682 positions, we found 14 editing sites in the $24 \mathrm{M}$ group and 41 editing sites in $29 \mathrm{M}$ that showed significant change in editing frequency, corresponding to 12 and 35 genes, respectively (Figure 5A, Table S8). Interestingly, when we compared genes that undergo altered RNA editing in the aging hippocampus to the list of differentially expressed and spliced genes, there was almost no overlap (Figure 5B) suggesting that gene expression, splicing and RNA editing control 
A

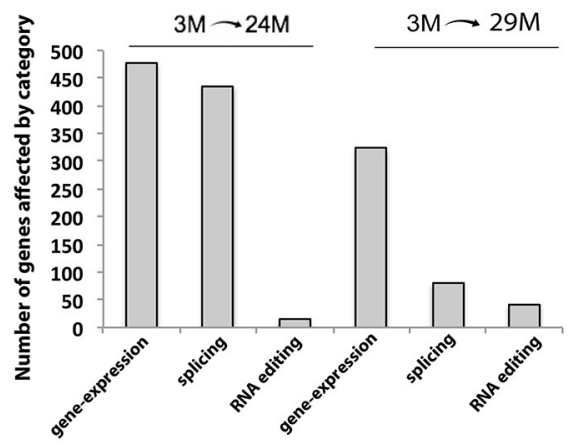

C

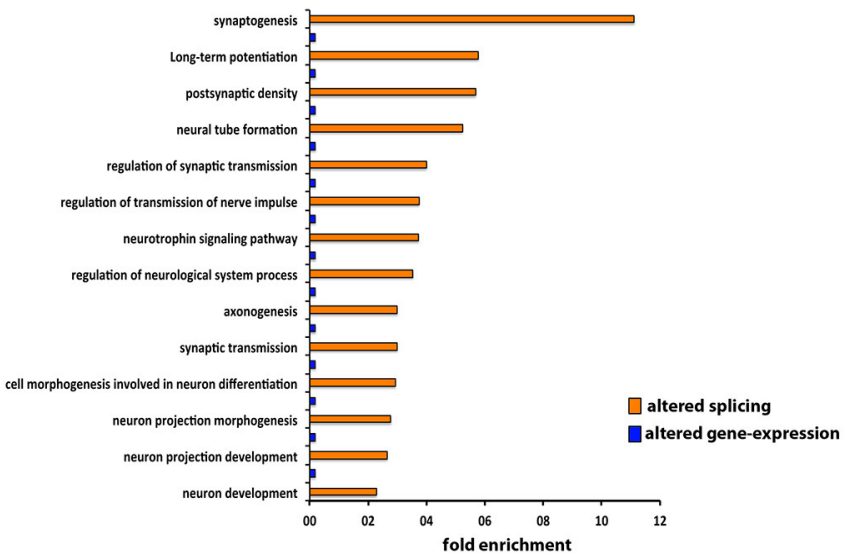

B

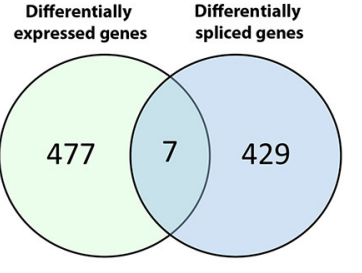

$3 \mathrm{M} \sim 24 \mathrm{M}$

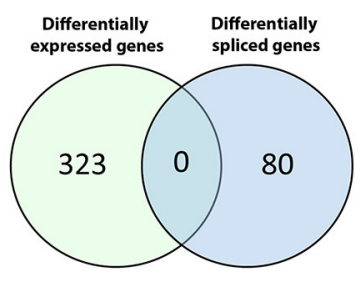

$3 \mathrm{M} \longrightarrow 29 \mathrm{M}$

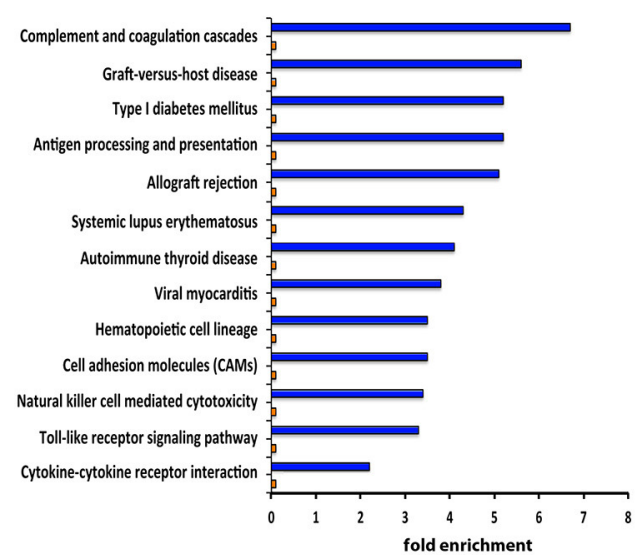

D

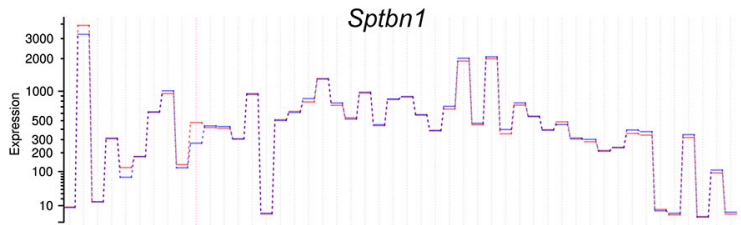

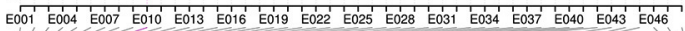

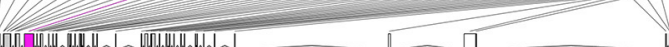

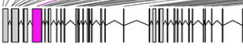

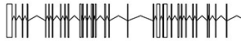

[I]

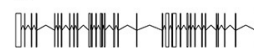

.

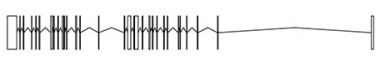

$\|$

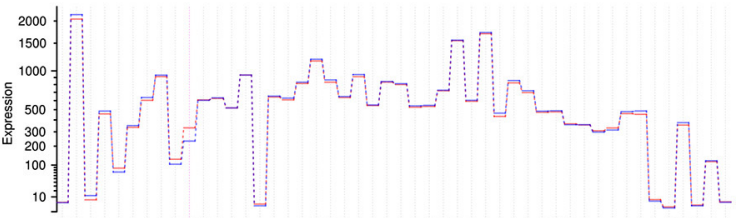

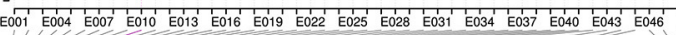

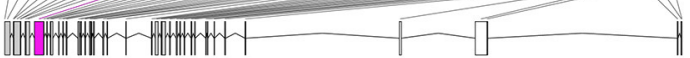

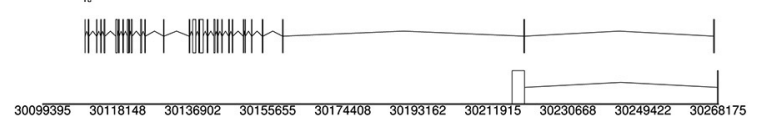

FIGURE 4 | Differential exon usage and alternative splicing changes. (A) Number of hippocampal genes affected by differential expression, splicing or RNA-editing during aging. (B) Venn-diagrams showing the overlap of genes affected by expression and/or splicing between age groups. Note that there is little to no overlap. (C) Cellular pathways affected in 3- vs. 24/29-month-old mice by splicing or expression levels. The data on differential expression is based on all up- or down-regulated genes. (D) One of the overlapping genes was Spectrin $\beta$, non-erythrocytic 1 (Sptbn1), showing higher expression of exon 10 (pink box) in aged mice, suggesting higher abundance of the transcript isoform 2 (see Figure S2 for details on isoform and domain structure). 
A

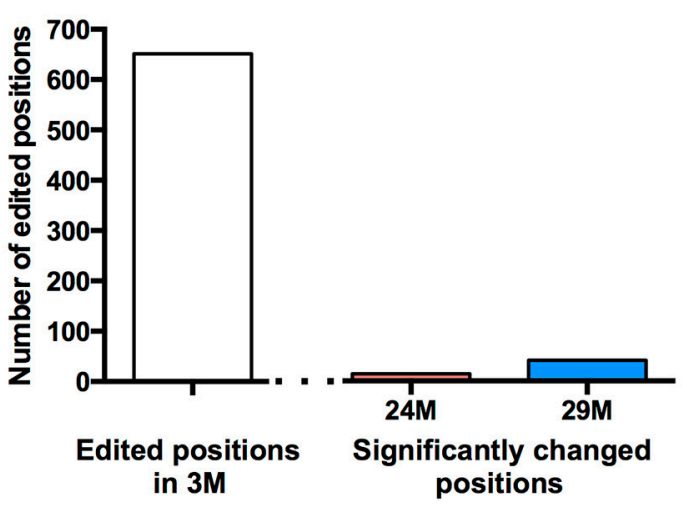

C

Pisd-ps1 pseudogene transcript

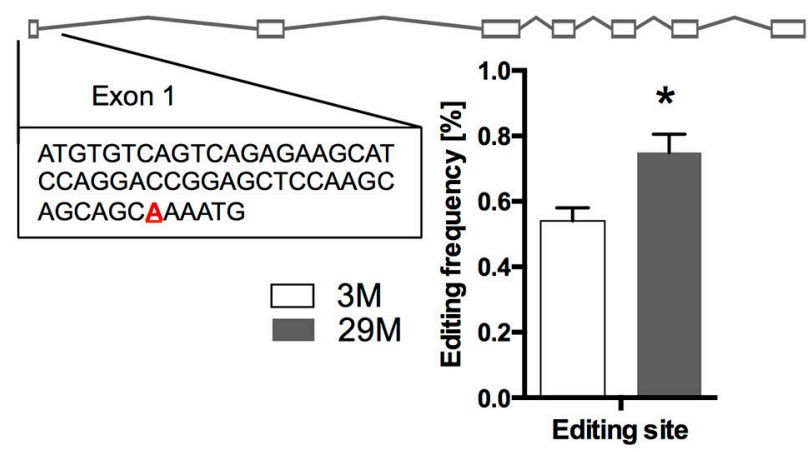

B

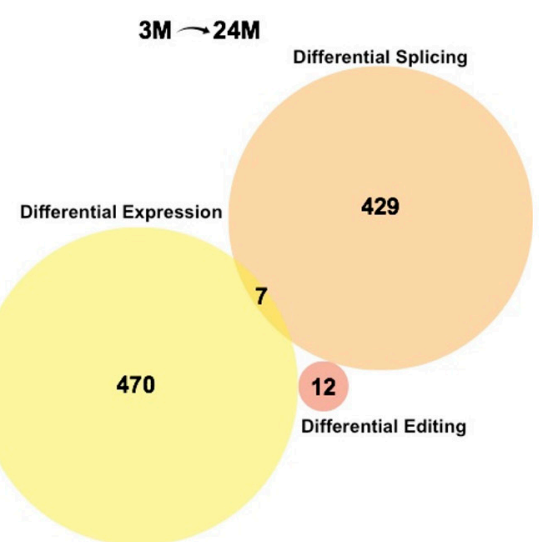

$3 \mathrm{M} \frown 29 \mathrm{M}$

D

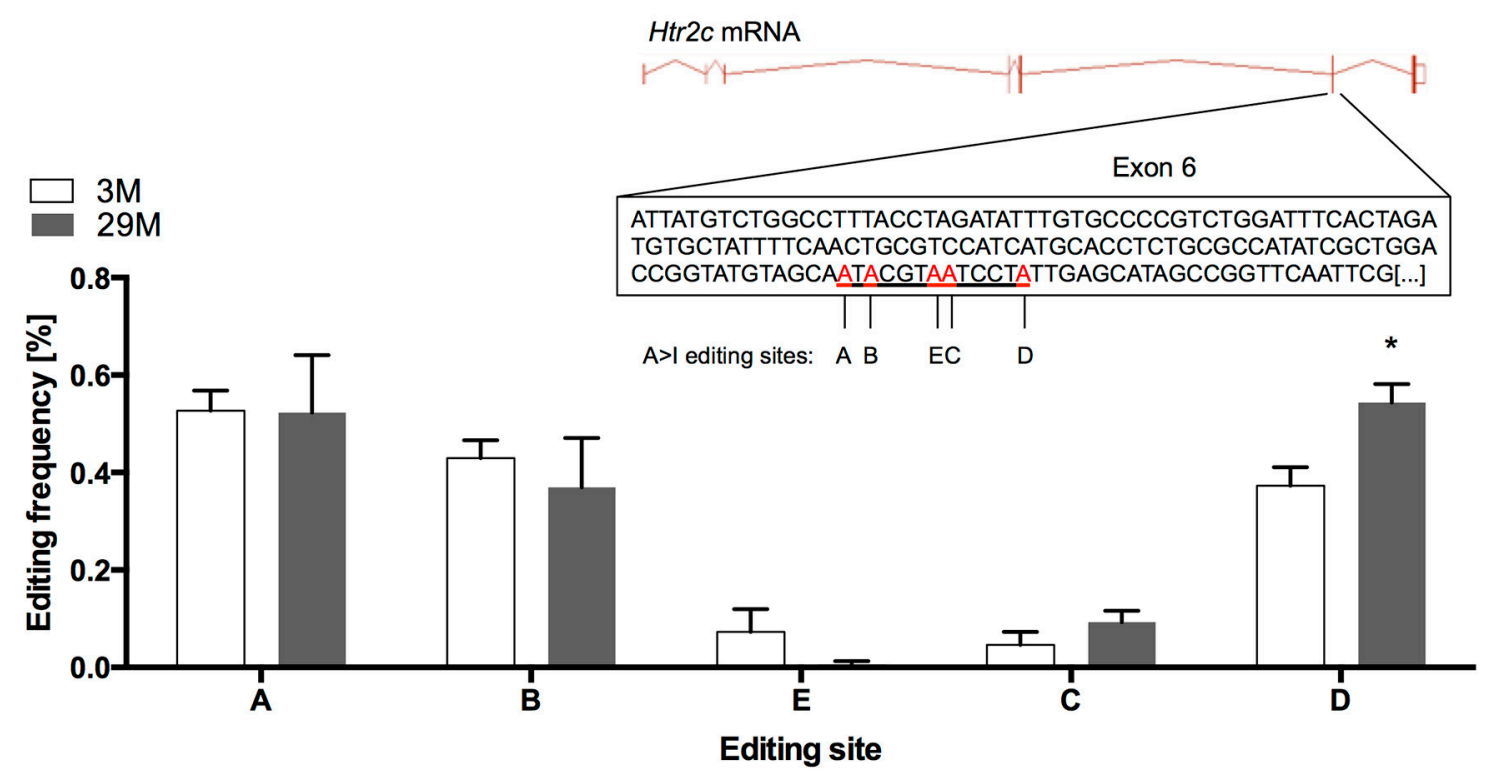

FIGURE 5 | RNA editing in the aging hippocampus. (A) Total number of RNA-editing events detectable in the hippocampus of 3-month-old mice and number of changes occurring during aging. (B) Venn diagram showing the overlap of differentially expressed genes, genes affected by splicing and RNA-editing in 24-month-old mice (upper panel) and 29-month-old mice (lower panel). (C) Editing of the pseudogene-transcribed ncRNA Pisd-ps 1 within exon 1 was found to be significantly increased in the $29 \mathrm{M}$ group (D) Within the well-described editing cassette of the Htr2c mRNA, editing was significantly upregulated at position $\mathrm{D}\left({ }^{*} p<0.05, n=3\right.$ per group). Error bars indicate s.e.m. 
distinct cellular pathways in the aging hippocampus. Only one of the genes that was characterized by altered RNA editing in 29-month-old mice was also up-regulated at the gene expression level (Figure 5C). This gene (Pisd-ps1) codes for a non-coding RNA expressed from a pseudogene with unknown function.

We also found increased editing of the well-established RNAediting target serotonin receptor $2 c$ (Htr $2 c$ ) (Figure 5D). It has been shown that increased editing of the Htr2c mRNA leads to a decrease in the receptor's signaling fidelity, which is in line with declining function of the serotonergic system with aging (Fidalgo et al., 2013). Moreover, altered RNA-editing of the Htr2c gene was observed in a mouse model for impaired memory function (Stilling et al., 2014).

Another gene that showed higher editing frequency with aging was Slc7a2, also known as Cat2 or Ctn. Interestingly, it has been described in the literature that the Ctn isoform of this gene undergoes nuclear retention and becomes part of paraspeckles (Bond and Fox, 2009), supporting the previous observation that aging is accompanied by increased nuclear paraspeckle formation.

In summary, our results suggest that hippocampal aging correlates to different degrees with altered gene expression, RNA splicing and RNA editing. Of note all of these processes appear to specifically account for age-associated changes in distinct cellular pathways. Namely, the induction of inflammatory processes is linked to altered gene expression while decline of synaptic plasticity seems to be mainly due to differential splicing.

\section{DISCUSSION}

In the present study we performed RNA sequencing in the hippocampus of three different aging groups to determine differential gene expression, exon usage and RNA-editing. Though brain aging has previously been described to go along with transcriptional changes, a detailed, homogenous picture of the transcriptome of the aging mouse hippocampus, especially toward the end of an individual's life span using RNA sequencing, has not been drawn yet.

Several studies have used microarray approaches to investigate age-associated transcriptional profiles of different brain regions in different species and came to heterogeneous results (Finch and Morgan, 1990; Pletcher et al., 2002; Blalock et al., 2003, 2010; Lu et al., 2004; Verbitsky et al., 2004; Xu et al., 2007; Zahn et al., 2007; Loerch et al., 2008; Pawlowski et al., 2009; Bishop et al., 2010), which may in part reflect differences in the technology available and also limited information on the mouse genome and transcriptome at the time the corresponding studies were conducted.

When we analyzed differential gene expression among the different age groups, the most striking effect was the up-regulation of genes linked to inflammatory processes with the complement system being the top ranking pathway identified by geneset enrichment, as well as pathway analysis using the Kyoto Encyclopedia of Genes and Genomes (KEGG) database. Increased neuroinflammation is likely the result of multiple up-stream factors such as impaired blood-brain barrier function (Lynch and Johnson, 2012; Erickson and Banks, 2013) leading to increased invasion of peripheral bacteria and viruses as well as cells of the peripheral immune system, which will cause a defense response against these invaders (Blau et al., 2012; Marques et al., 2013). Moreover, increased clearance of damaged or misfolded proteins and other cellular debris becomes necessary with increasing age and these aggregates will activate the immune system (predominantly microglia) (Fonseca et al., 2004; Czirr and Wyss-Coray, 2012).

Of note, up-regulation of neuroinflammatory genes with aging clearly delivers an explanation why neurodegenerative diseases such as $\mathrm{AD}$ become increasingly abundant with advanced age. Based on our findings, we derive the hypothesis that diseaserelated mutations become only detrimental when the respective genes become expressed or activated. As such, aging is the strongest risk factor for AD (Lopez, 2011). The reason why lateonset $\mathrm{AD}$ (LOAD) is associated with increasing age is not yet fully understood. However, accumulating evidence and a plethora of genome-wide association studies (GWAS) link LOAD to a fast growing number of genetic risk factors and it seems feasible that these genetic risk factors result in LOAD due to changes at the transcriptome and proteome level. Indeed, in our hippocampal aging study in mice, we found up-regulation of several homologs, interaction partners or other closely related genes to almost all genes that appear to be the top 10 single genetic risk factors for LOAD as designated by the AlzGene database (Bertram et al., 2007). Of particular interest in light of our data is the gene coding for complement receptor 1 (CR1), since this receptor binds processed $\mathrm{C} 4$ and $\mathrm{C} 3$ complement proteins including the products of the $C 4 b$ gene, which we found to be most prominently up-regulated in the aging hippocampus. Single nucleotide polymorphisms (SNPs) and copy number variations (CNVs) in the human CR1 gene were found to be highly associated with late onset $\mathrm{AD}$ in a number of studies and across ethnic groups (Bertram et al., 2007; Lambert et al., 2009; Biffi et al., 2010, 2012; Carrasquillo et al., 2010; Chibnik et al., 2011; Brouwers et al., 2012; Crehan et al., 2012; Hazrati et al., 2012; Keenan et al., 2012; Ma et al., 2014). This makes it very tempting to draw the conclusion that increased expression of C3 and C4 with aging will result in aberrant regulation of the complement cascade, which in turn lead to neurodegeneration in carriers of the "wrong" CR1 allele. Aberrant regulation could lead to neurodegeneration in two different, non-mutual exclusive ways. On the one hand, differential CR1 regulatory activity could lead to less effective $A \beta$ aggregate clearance (Fonseca et al., 2004). On the other hand, de-regulated activation of the complement system could result in collateral damage by overactive inflammation (reviewed in Czirr and Wyss-Coray, 2012). Along this line, CR1 inhibition was shown to prevent microglia activation (Crehan et al., 2013). Of note, mice do not express CR1 but mice that lack the murine ortholog Cr1-related protein Y (Crry) show reduced inflammatory responses and attenuated increases in AD-related disease progress biomarkers (Killick et al., 2013), further suggesting that activation of the complement system is a key feature of the aging hippocampus that also plays a role in $\mathrm{AD}$ pathogenesis.

The exact mechanism of altered CR1 regulatory function in this complex network is still to be elucidated but a beneficial effect of complement inhibition in general has been demonstrated (Rancan et al., 2003; Fonseca et al., 2004; Leinhase et al., 
2006; Kulkarni et al., 2008, 2011; Pillay et al., 2008), yet this data remains controversial (Wyss-Coray et al., 2002; Loeffler, 2004; Maier et al., 2008). Nevertheless, our data support the view that targeting the complement system and other inflammatory pathways poses an intriguing possibility for the treatment of agingassociated diseases and cognitive decline, which has recently been demonstrated for inhibition of NF- $\mathrm{KB}$ signaling in a mouse model of $\mathrm{AD}$ (Liu et al., 2014). Likewise, physical activity in rodents and humans has been shown to improve cognitive abilities during aging (van Praag et al., 1999; Praag et al., 2005; Erickson et al., 2011). Remarkably, a recent study could show in aged mice that voluntary wheel running leads to a reduction in C4b expression in the hippocampus (Kohman et al., 2011), demonstrating a clear correlation between $\mathrm{C} 4$ levels and cognitive abilities, which is further supported by the finding that $\mathrm{C} 4$ inhibition by external application of a vaccinia virus complement control protein has beneficial effects on memory performance in mouse models of AD (Kulkarni et al., 2008, 2011).

While it is not entirely clear if the age-associated induction of immune genes is cause or consequence of altered glia cell function that arise on the background of altered activity and/or increased cell number (Lolova, 1991; Amenta et al., 1998; Long et al., 1999; Blalock et al., 2003; Takahashi et al., 2006; Hayakawa et al., 2007) astrocytes and especially microglia are clearly good candidates for a potent source of immune system molecules (Hosokawa et al., 2003). We did not observe significant changes in the number of neurons, astrocytes or microglia in our study. In line with this, the corresponding marker genes for neurons and microglia were not altered in our gene-expression analysis. The astrocyte marker gene gfap was however increased during aging, indicating that the inflammatory response in our study in mainly linked to a change in the active state of glia cells. However, also neurons are known to secrete complement proteins (Shen et al., 1997; Terai et al., 1997; Hosokawa et al., 2003) and future studies should therefore also focus on untangling which cell types are the main contributors to the observed neuroinflammatory program. In any case $C 4 b$ is an interesting gene, since its expression strongly correlated with age and it was in fact one of the most significant changes that have been reported by others and within this study. It was already found to be up-regulated as one of the very few DEGs in the comparison of hippocampal expression in 3-monthold mice with 16-month-old mice (3-fold up-regulation) (Peleg et al., 2010). Interestingly, out of the 12 up-regulated genes found in this independent study, 6 genes were also found up-regulated in the present study. Along with $C 4 b$ and $C 3$-another complement component frequently found up-regulated-these were 1700112E06Rik, BC061194, Cox8b and Sult1c2. Hence, these 6 genes obviously belong to a set of genes that are highly associated with murine hippocampal aging. In addition, other studies have observed complement-gene up-regulation in studies of aging in the hippocampus as well as in the prefrontal cortex (Verbitsky et al., 2004; Reichwald et al., 2009; Bordner et al., 2011; Stephan et al., 2013).

To our knowledge, the present study is the first to also specifically report the role of long-non-coding RNAs in the aging hippocampus. We found several long-non-coding RNAs to be differentially expressed. Little is known so far on the role of these RNAs, but the up-regulation of Neat 1 and the associated ncRNA Malat1 was interesting since Malat1 is highly abundant in neurons and has been associated with the regulation of gene expression and splicing, but was also found to affect the expression of genes linked to synaptic function (Bernard et al., 2010). Of note, a previous study reported that Malat 1 is increased in the hippocampus of alcoholics (Kryger et al., 2012), a condition clearly linked to cognitive impairment. Another interesting finding was the up-regulation of Pisd-ps1. Though the Pisd-ps1 ncRNA has no annotated function so far, it has been described to be up-regulated with aging (Bordner et al., 2011; Sousa-Victor et al., 2014). Pisd-ps1 also harbors a known RNA-editing site and was one of the few transcripts that show a higher editing frequency in 29-month-old mice. Thus, this ncRNA might be implicated in brain aging and warrants further research on its regulatory functions.

To obtain more insight on the mechanisms that drive ageassociated changes in hippocampal gene expression, we analyzed the promoters of the differentially regulated transcripts. We found high enrichment of TFBS involved in pro-inflammatory signaling cascades. This trend was even more pronounced in 29-month-old mice, which supports our interpretation of increasing neuroinflammation with advancing age as part of the aging program. One of the key factors contributing to the neuroinflammatory gene expression response in the aging hippocampus was STAT1. Interestingly, mice that lack STAT1 show enhanced memory performance and are resistant to memory impairment induced by $A B$ peptides injected into the hippocampus (Hsu et al., 2014). Thus, activation of STAT1 might be a key step in age-associated memory impairment.

RNA sequencing also allows the analysis of differential splicing events and previous data suggest that differential exon usage undergoes substantial changes during brain development (Tollervey et al., 2011; Mazin et al., 2013). Differential splicing has also been observed in postmortem brain tissue from patients that suffered from sporadic AD (Twine et al., 2011; Mills et al., 2013) and in mouse models for amyloid deposition (Kim et al., 2012). This data is in line with increased protein levels of spliceosome components in AD patients (Bai et al., 2013). However, RNA sequencing has not been used to analyze changes in the aging hippocampus as described in this study. The first interesting observation is that massive changes in RNA splicing occur when we compared the hippocampus of 3-month-old mice to the 24- and 29-month groups. Another highly interesting observation was that there was little to no overlap among the genes differentially expressed and the genes that were differentially spliced during aging. The most striking finding is, however, that genes affected by age-associated changes in splicing were associated with neuronal and synaptic function, including neurite outgrowth and LTP. This is in line with the well-described decline in synaptic plasticity with aging (Blau et al., 2012). A number of interesting candidate genes were identified and await further analysis. For example, we identified Spectrin $\beta$, non-erythrocytic 1 (Sptbn1) to be differentially spliced during aging. Sptbn1 is involved in tethering the actin cytoskeleton to the plasma membrane, which is necessary for neurite outgrowth and possibly spine morphology and motility (Lee et al., 2012). Interestingly, introduction of the 
observed exon change in this gene may indicate a shift toward an isoform that cannot perform this function anymore, since it lacks the essential membrane-tethering $\mathrm{PH}$ protein domain. Together, these results confirm findings in the literature and make differential exon usage analysis a plausible tool for the study of age-related changes in the transcriptome. The fact that the age-associated inflammatory response is driven by changes in gene expression that could be linked to a number of key transcription factors while genes linked to synaptic plasticity are mainly affected by alternative splicing also suggests that functionally distinct gene expression programs in the adult brain are regulated by specific mechanisms.

In conclusion, our data deciphers at an unprecedented depth the hippocampal transcriptional program linked to aging. While changes in the absolute levels of RNA transcripts are linked to an inflammatory response and especially activation of the complement system, changes in alterative splicing affect genes linked to synaptic plasticity. At the same time RNA editing does not appear to play a major, genome-wide role in the aging hippocampus. This data provides important novel information, and multiple starting points for further analysis of potential therapeutic interventions and strongly suggests a causal relationship between aging-dependent changes in gene expression and the late onset of neurodegenerative diseases, most prominently AD.

\section{ACKNOWLEDGMENTS}

We thank Dr. Pooja Rao for helpful comments on data analysis and interpretation. This project was partly supported by the following funds to Andre Fischer: the Euryi award (DFG grant FI 981), the ERA NET Neuron Project EPITHERAPY, the Schram Foundation, the Hans and Ilse Breuer Foundation and the German Center for Neurodegenerative Diseases (DZNE) Göttingen.

\section{SUPPLEMENTARY MATERIAL}

The Supplementary Material for this article can be found online at: http://www.frontiersin.org/journal/10.3389/fncel.2014. 00373/abstract

Figure S1 | Immunohistochemical analysis of hippocampal cell number. (A) Representative images showing immunostaining for the Neuronal maker protein Neuronal N (NeuN), the Microglia marker lonized calcium binding adaptor molecule 1 (IBA1) and the astrocyte marker Glial fibrillary acidic protein (GFAP) in 3 and 29-month old mice. (B) Quantification of A. CA1; hippocampal subregion CA1, cc; corpus calosum. Scale bar: $100 \mu \mathrm{m}$.

Figure S2 | Functionally enriched pathway in 3 vs. 24 and 29 month old mice identified on the basis of gene array data. Left panel: Enriched pathways identified on the basis of differentially expressed genes comparing hippocampal RNA from 3- vs. 24-month-old mice. Right panel: Enriched pathways identified on the basis of differentially expressed genes comparing hippocampal RNA from 3 vs. 29-month-old mice.

Figure S3 | Genetic architecture and domain structure of Sptbn1. The figure was downloaded from the ENSEMBLE database (version e76). It shows the genomic locus of Sptbn 1 and its annotated transcribed isoforms including exon structure and encoded protein domains. Isoform 2 is marked in yellow. This was the isoform that is likely underlying the switch in exon usage detected in RNA-seq data in $24 \mathrm{M}$ and $29 \mathrm{M}$ groups.

\section{REFERENCES}

Agis-Balboa, R. C., Arcos-Diaz, D., Wittnam, J., Govindarajan, N., Blom, K., Burkhardt, S., et al. (2011). A hippocampal insulin-growth factor 2 pathway regulates the extinction of fear memories. EMBO J. 30, 4071-4083. doi: 10.1038/emboj.2011.293

Amenta, F., Bronzetti, E., Sabbatini, M., and Vega, J. A. (1998). Astrocyte changes in aging cerebral cortex and hippocampus: a quantitative immunohistochemical study. Microsc. Res. Tech. 43, 29-33.

Anders, S., and Huber, W. (2010). Differential expression analysis for sequence count data. Genome Biol. 11:R106. doi: 10.1186/gb-2010-11-10-r106

Anders, S., Reyes, A., and Huber, W. (2012). Detecting differential usage of exons from RNA-seq data. Genome Res. 22, 2008-2017. doi: 10.1101/gr.133744.111

Antunes, M., and Biala, G. (2012). The novel object recognition memory: neurobiology, test procedure, and its modifications. Cogn. Process. 13, 93-110. doi: 10.1007/s10339-011-0430-z

Bai, B., Hales, C. M., Chen, P. C., Gozal, Y., Dammer, E. B., Fritz, J. J., et al. (2013). U1 small nuclear ribonucleoprotein complex and RNA splicing alterations in Alzheimer's disease. Proc. Natl. Acad. Sci. U.S.A. 110, 16562-16567. doi: 10.1073/pnas.1310249110

Benjamini, Y., and Hochberg, Y. (1995). Controlling the false discovery rate - a practical and powerful approach to multiple testing. J. R. Stat. Soc B Methodol. 57, 289-300.

Berchtold, N. C., Cribbs, D. H., Coleman, P. D., Rogers, J., Head, E., Kim, R., et al. (2008). Gene expression changes in the course of normal brain aging are sexually dimorphic. Proc. Natl. Acad. Sci. U.S.A. 105, 15605-15610. doi: 10.1073/pnas.0806883105

Bernard, D., Prasanth, K. V., Tripathi, V., Colasse, S., Nakamura, T., Xuan, Z., et al. (2010). A long nuclear-retained non-coding RNA regulates synaptogenesis by modulating gene expression. EMBO J. 29, 3082-3093. doi: 10.1038/emboj.2010.199

Bertram, L., McQueen, M. B., Mullin, K., Blacker, D., and Tanzi, R. E. (2007). Systematic meta-analyses of Alzheimer disease genetic association studies: the AlzGene database. Nat. Genet. 39, 17-23. doi: 10.1038/ ng1934

Biffi, A., Anderson, C. D., Desikan, R. S., Sabuncu, M., Cortellini, L., Schmansky, N., et al. (2010). Genetic variation and neuroimaging measures in Alzheimer disease. Arch. Neurol. 67, 677-685. doi: 10.1001/archneurol.20 10.108

Biffi, A., Shulman, J. M., Jagiella, J. M., Cortellini, L., Ayres, A. M., Schwab, K., et al. (2012). Genetic variation at CR1 increases risk of cerebral amyloid angiopathy. Neurology 78, 334-341. doi: 10.1212/WNL.0b013e31824 $52 \mathrm{~b} 40$

Bishop, N. A., Lu, T., and Yankner, B. A. (2010). Neural mechanisms of ageing and cognitive decline. Nature 464, 529-535. doi: 10.1038/nature 08983

Blalock, E. M., Chen, K. C., Sharrow, K., Herman, J. P., Porter, N. M., Foster, T. C., et al. (2003). Gene microarrays in hippocampal aging: statistical profiling identifies novel processes correlated with cognitive impairment. J. Neurosci. 23, 3807-3819.

Blalock, E. M., Grondin, R., Chen, K. C., Thibault, O., Thibault, V., Pandya, J. D., et al. (2010). Aging-related gene expression in hippocampus proper compared with dentate gyrus is selectively associated with metabolic syndrome variables in rhesus monkeys. J. Neurosci. 30, 6058-6071. doi: 10.1523/JNEUROSCI.395609.2010

Blau, C. W., Cowley, T. R., O’Sullivan, J., Grehan, B., Browne, T. C., Kelly, L., et al. (2012). The age-related deficit in LTP is associated with changes in perfusion and blood-brain barrier permeability. Neurobiol. Aging 33, 1005.e23-1005.e35. doi: 10.1016/j.neurobiolaging.2011.09.035

Bond, C. S., and Fox, A. H. (2009). Paraspeckles: nuclear bodies built on long noncoding RNA. J. Cell Biol. 186, 637-644. doi: 10.1083/jcb.200906113

Bordner, K. A., Kitchen, R. R., Carlyle, B., George, E. D., Mahajan, M. C., Mane, S. M., et al. (2011). Parallel declines in cognition, motivation, and locomotion in aging mice: association with immune gene upregulation in the medial prefrontal cortex. Exp. Gerontol. 46, 643-659. doi: 10.1016/j.exger.2011.03.003

Broadbent, N. J., Gaskin, S., Squire, L. R., and Clark, R. E. (2010). Object recognition memory and the rodent hippocampus. Learn. Mem. 17, 5-11. doi: 10.1101/lm.1650110

Brouwers, N., Van Cauwenberghe, C., Engelborghs, S., Lambert, J. C., Bettens, K., Le Bastard, N., et al. (2012). Alzheimer risk associated with a copy number 
variation in the complement receptor 1 increasing C3b/C4b binding sites. Mol. Psychiatry 17, 223-233. doi: 10.1038/mp.2011.24

Carrasquillo, M. M., Belbin, O., Hunter, T. A., Ma, L., Bisceglio, G. D., Zou, F, et al. (2010). Replication of CLU, CR1, and PICALM associations with Alzheimer disease. Arch. Neurol. 67, 961-964. doi: 10.1001/archneurol.20 10.147

Chibnik, L. B., Shulman, J. M., Leurgans, S. E., Schneider, J. A., Wilson, R. S., Tran, D., et al. (2011). CR1 is associated with amyloid plaque burden and age-related cognitive decline. Ann. Neurol. 69, 560-569. doi: 10.1002/ana. 22277

Crehan, H., Hardy, J., and Pocock, J. (2013). Blockage of CR1 prevents activation of rodent microglia. Neurobiol. Dis. 54, 139-149. doi: 10.1016/j.nbd.2013. 02.003

Crehan, H., Holton, P., Wray, S., Pocock, J., Guerreiro, R., and Hardy, J. (2012). Complement receptor 1 (CR1) and Alzheimer's disease. Immunobiology 217, 244-250. doi: 10.1016/j.imbio.2011.07.017

Czirr, E., and Wyss-Coray, T. (2012). The immunology of neurodegeneration. J. Clin. Invest. 122, 1156-1163. doi: 10.1172/JCI58656

Dillman, A. A., Hauser, D. N., Gibbs, J. R., Nalls, M. A., McCoy, M. K., Rudenko, I. N., et al. (2013). mRNA expression, splicing and editing in the embryonic and adult mouse cerebral cortex. Nat. Neurosci. 16, 499-506. doi: 10.1038/ nn.3332

Dobin, A., Davis, C. A., Schlesinger, F., Drenkow, J., Zaleski, C., Jha, S., et al. (2013). STAR: ultrafast universal RNA-seq aligner. Bioinformatics 29, 15-21. doi: 10.1093/bioinformatics/bts635

Erickson, K. I., Voss, M. W., Prakash, R. S., Basak, C., Szabo, A., Chaddock, L., et al. (2011). Exercise training increases size of hippocampus and improves memory. Proc. Natl. Acad. Sci. U.S.A. 108, 3017-3022. doi: 10.1073/pnas.1015950108

Erickson, M. A., and Banks, W. A. (2013). Blood-brain barrier dysfunction as a cause and consequence of Alzheimer's disease. J. Cereb. Blood Flow Metab. 33, 1500-1513. doi: 10.1038/jcbfm.2013.135

Fanselow, M. S. (2010). From contextual fear to a dynamic view of memory systems. Trends Cogn. Sci. 14, 7-15. doi: 10.1016/j.tics.2009.10.008

Fidalgo, S., Ivanov, D. K., and Wood, S. H. (2013). Serotonin: from top to bottom. Biogerontology 14, 21-45. doi: 10.1007/s10522-012-9406-3

Finch, C. E., and Morgan, D. G. (1990). RNA and protein metabolism in the aging brain. Annu. Rev. Neurosci. 13, 75-88. doi: 10.1146/annurev.ne.13.030190.000451

Fischer, A. (2014). Epigenetic memory: the Lamarckian brain. EMBO J. 33, 945-967. doi: 10.1002/embj.201387637

Fonseca, M. I., Zhou, J., Botto, M., and Tenner, A. J. (2004). Absence of C1q leads to less neuropathology in transgenic mouse models of Alzheimer's disease. J. Neurosci. 24, 6457-6465. doi: 10.1523/JNEUROSCI.0901-04.2004

Gentleman, R. C., Carey, V. J., Bates, D. M., Bolstad, B., Dettling, M., Dudoit, S., et al. (2004). Bioconductor: open software development for computational biology and bioinformatics. Genome Biol. 5, R80. doi: 10.1186/gb-2004-5-10-r80

Hayakawa, N., Kato, H., and Araki, T. (2007). Age-related changes of astorocytes, oligodendrocytes and microglia in the mouse hippocampal CA1 sector. Mech. Ageing Dev. 128, 311-316. doi: 10.1016/j.mad.2007.01.005

Hazrati, L.-N., Van Cauwenberghe, C., Brooks, P. L., Brouwers, N., Ghani, M., Sato, C., et al. (2012). Genetic association of CR1 with Alzheimer's disease: a tentative disease mechanism. Neurobiol. Aging 33, 2949.e5-2949.e12. doi: 10.1016/j.neurobiolaging.2012.07.001

Horiuchi, J., and Saitoe, M. (2005). Can flies shed light on our own age-related memory impairment? Ageing Res. Rev. 4, 83-101. doi: 10.1016/j.arr.2004. 10.001

Hosokawa, M., Klegeris, A., Maguire, J., and McGeer, P. L. (2003). Expression of complement messenger RNAs and proteins by human oligodendroglial cells. Glia 42, 417-423. doi: 10.1002/glia.10234

Hsu, W. L., Ma, Y. L., Hsieh, D. Y., Liu, Y. C., and Lee, E. H. (2014). STAT1 negatively regulates spatial memory formation and mediates the memory-impairing effect of Abeta. Neuropsychopharmacology 39, 746-758. doi: 10.1038/npp.20 13.263

Huang, D. W., Sherman, B. T., and Lempicki, R. A. (2009a). Bioinformatics enrichment tools: paths toward the comprehensive functional analysis of large gene lists. Nucleic Acids Res. 37, 1-13. doi: 10.1093/nar/gkn923

Huang, D. W., Sherman, B. T., and Lempicki, R. A. (2009b). Systematic and integrative analysis of large gene lists using DAVID bioinformatics resources. Nat. Protoc. 4, 44-57. doi: 10.1038/nprot.2008.211
Huber, W., von Heydebreck, A., Sültmann, H., Poustka, A., and Vingron, M. (2002). Variance stabilization applied to microarray data calibration and to the quantification of differential expression. Bioinformatics 18(Suppl. 1), S96-S104. doi: 10.1093/bioinformatics/18.suppl_1.S96

Jucker, M., and Ingram, D. K. (1997). Murine models of brain aging and age-related neurodegenerative diseases. Behav. Brain Res. 85, 1-26. doi: 10.1016/S01664328(96)02243-7

Keenan, B. T., Shulman, J. M., Chibnik, L. B., Raj, T., Tran, D., Sabuncu, M. R., et al. (2012). A coding variant in CR1 interacts with APOE- 44 to influence cognitive decline. Hum. Mol. Genet. 21, 2377-2388. doi: 10.1093/hmg/ dds054

Kerimoglu, C., Agis-Balboa, R. C., Kranz, A., Stilling, R., Bahari-Javan, S., Benito-Garagorri, E., et al. (2013). Histone-methyltransferase mll2 (kmt2b) is required for memory formation in mice. J. Neurosci. 33, 3452-3464. doi: 10.1523/JNEUROSCI.3356-12.2013

Killick, R., Hughes, T. R., Morgan, B. P., and Lovestone, S. (2013). Deletion of Crry, the murine ortholog of the sporadic Alzheimer's disease risk gene CR1, impacts tau phosphorylation and brain CFH. Neurosci. Lett. 533, 96-99. doi: 10.1016/j.neulet.2012.11.008

Kim, K. H., Moon, M., Yu, S. B., Mook-Jung, I., and Kim, J. I. (2012). RNASeq analysis of frontal cortex and cerebellum from 5XFAD mice at early stage of disease pathology. J. Alzheimers Dis. 29, 793-808. doi: 10.3233/JAD-2012111793

Kohman, R. A., Rodriguez-Zas, S. L., Southey, B. R., Kelley, K. W., Dantzer, R., and Rhodes, J. S. (2011). Voluntary wheel running reverses age-induced changes in hippocampal gene expression. PLoS ONE 6:e22654. doi: 10.1371/journal.pone.0022654

Koivisto, K., Reinikainen, K. J., Hanninen, T., Vanhanen, M., Helkala, E. L., Mykkanen, L., et al. (1995). Prevalence of age-associated memory impairment in a randomly selected population from eastern Finland. Neurology 45, 741-747. doi: 10.1212/WNL.45.4.741

Kryger, R., Fan, L., Wilce, P. A., and Jaquet, V. (2012). MALAT-1, a non protein-coding RNA is upregulated in the cerebellum, hippocampus and brain stem of human alcoholics. Alcohol 46, 629-634. doi: 10.1016/j.alcohol.2012. 04.002

Kulkarni, A. P., Govender, D. A., Kotwal, G. J., and Kellaway, L. A. (2011). Modulation of anxiety behavior by intranasally administered vaccinia virus complement control protein and curcumin in a mouse model of Alzheimer's disease. Curr. Alzheimer Res. 8, 95-113. doi: 10.2174/1567205117946 04598

Kulkarni, A. P., Pillay, N. S., Kellaway, L. A., and Kotwal, G. J. (2008). Intracranial administration of vaccinia virus complement control protein in $\mathrm{Mo} / \mathrm{Hu}$ APPswe PS1dE9 transgenic mice at an early age shows enhanced performance at a later age using a cheese board maze test. Biogerontology 9, 405-420. doi: 10.1007/s10522-008-9161-7

Lambert, J.-C., Heath, S., Even, G., Campion, D., Sleegers, K., Hiltunen, M., et al. (2009). Genome-wide association study identifies variants at CLU and CR1 associated with Alzheimer's disease. Nat. Genet. 41, 1094-1099. doi: $10.1038 / \mathrm{ng} .439$

Lee, H. J., Lee, K., and Im, H. (2012). alpha-Synuclein modulates neurite outgrowth by interacting with SPTBN1. Biochem. Biophys. Res. Commun. 424, 497-502. doi: 10.1016/j.bbrc.2012.06.143

Leinhase, I., Schmidt, O. I., Thurman, J. M., Hossini, A. M., Rozanski, M., Taha, M. E., et al. (2006). Pharmacological complement inhibition at the C3 convertase level promotes neuronal survival, neuroprotective intracerebral gene expression, and neurological outcome after traumatic brain injury. Exp. Neurol. 199, 454-464. doi: 10.1016/j.expneurol.2006.01.033

Liu, Y., Liu, X., Hao, W., Decker, Y., Schomburg, R., Fulop, L., et al. (2014). IKKbeta deficiency in myeloid cells ameliorates Alzheimer's disease-related symptoms and pathology. J. Neurosci. 34, 12982-12999. doi: 10.1523/JNEUROSCI.134814.2014

Loeffler, D. A. (2004). Using animal models to determine the significance of complement activation in Alzheimer's disease. J. Neuroinflammation 1:18. doi: 10.1186/1742-2094-1-18

Loerch, P. M., Lu, T., Dakin, K. A., Vann, J. M., Isaacs, A., Geula, C., et al. (2008). Evolution of the aging brain transcriptome and synaptic regulation. PLoS ONE 3:e3329. doi: 10.1371/journal.pone.0003329

Lolova, I. (1991). Qualitative and quantitative glial changes in the hippocampus of aged rats. Anat. Anz. 172, 263-271. 
Long, J. M., Mouton, P. R., Jucker, M., and Ingram, D. K. (1999). What counts in brain aging? Design-based stereological analysis of cell number. J. Gerontol. A Biol. Sci. Med. Sci. 54, B407-417. doi: 10.1093/gerona/54. $10 . \mathrm{B} 407$

Lopez, O. L. (2011). The growing burden of Alzheimer's disease. Am. J. Manag. Care 17(Suppl. 13), S339-S345.

Lu, T., Pan, Y., Kao, S.-Y., Li, C., Kohane, I., Chan, J., et al. (2004). Gene regulation and DNA damage in the ageing human brain. Nature 429, 883-891. doi: 10.1038/nature02661

Lynch, M. A., and Johnson, R. W. (2012). The impact of aging on the brain-risk, resilience and repair. Brain Behav. Immun. 26, 714-716. doi: 10.1016/j.bbi.2012.02.005

Ma, X. Y., Yu, J. T., Tan, M. S., Sun, F. R., Miao, D., and Tan, L. (2014). Missense variants in CR1 are associated with increased risk of Alzheimer' disease in Han Chinese. Neurobiol. Aging 35, 443.e17-443.e21. doi: 10.1016/j.neurobiolaging. 2013.08.009

Maier, M., Peng, Y., Jiang, L., Seabrook, T. J., Carroll, M. C., and Lemere, C. A. (2008). Complement C3 deficiency leads to accelerated amyloid beta plaque deposition and neurodegeneration and modulation of the microglia/macrophage phenotype in amyloid precursor protein transgenic mice. J. Neurosci. 28, 6333-6341. doi: 10.1523/JNEUROSCI.082908.2008

Marques, F., Sousa, J. C., Sousa, N., and Palha, J. A. (2013). Blood-brain-barriers in aging and in Alzheimer's disease. Mol. Neurodegener. 8:38. doi: 10.1186/17501326-8-38

Mazin, P., Xiong, J., Liu, X., Yan, Z., Zhang, X., Li, M., et al. (2013). Widespread splicing changes in human brain development and aging. Mol. Syst. Biol. 9:633. doi: $10.1038 / \mathrm{msb} .2012 .67$

Mills, J. D., Nalpathamkalam, T., Jacobs, H. I., Janitz, C., Merico, D., Hu, P., et al. (2013). RNA-Seq analysis of the parietal cortex in Alzheimer's disease reveals alternatively spliced isoforms related to lipid metabolism. Neurosci. Lett. 1, 90-95. doi: 10.1016/j.neulet.2012.12.042

Montesanto, A., Dato, S., Bellizzi, D., Rose, G., and Passarino, G. (2012). Epidemiological, genetic and epigenetic aspects of the research on healthy ageing and longevity. Immun. Ageing 9:6. doi: 10.1186/1742-49 33-9-6

Mootha, V. K., Lindgren, C. M., Eriksson, K. F., Subramanian, A., Sihag, S., Lehar, J., et al. (2003). PGC-1alpha-responsive genes involved in oxidative phosphorylation are coordinately downregulated in human diabetes. Nat. Genet. 34, 267-273. doi: 10.1038/ng1180

Pawlowski, T. L., Bellush, L. L., Wright, A. W., Walker, J. P., Colvin, R. A., and Huentelman, M. J. (2009). Hippocampal gene expression changes during age-related cognitive decline. Brain Res. 1256, 101-110. doi: 10.1016/j.brainres.2008.12.039

Peleg, S., Sananbenesi, F., Zovoilis, A., Burkhardt, S., Bahari-Javan, S., AgisBalboa, R. C., et al. (2010). Altered histone acetylation is associated with agedependent memory impairment in mice. Science 328, 753-756. doi: 10.1126/science.1186088

Peng, S. L. (2008). Transcription factors in autoimmune diseases. Front. Biosci. 13:4218-4240. doi: 10.2741/3001

Picardi, E., and Pesole, G. (2013). REDItools: high-throughput RNA editing detection made easy. Bioinformatics 29, 1813-1814. doi: 10.1093/bioinformatics/btt287

Pillay, N. S., Kellaway, L. A., and Kotwal, G. J. (2008). Early detection of memory deficits and memory improvement with vaccinia virus complement control protein in an Alzheimer's disease model. Behav. Brain Res. 192, 173-177. doi: 10.1016/j.bbr.2008.03.038

Pletcher, S. D., Macdonald, S. J., Marguerie, R., Certa, U., Stearns, S. C., Goldstein, D. B., et al. (2002). Genome-wide transcript profiles in aging and calorically restricted Drosophila melanogaster. Curr. Biol. 12, 712-723. doi: 10.1016/S0960-9822(02)00808-4

Praag, H. V., Shubert, T., Zhao, C., and Gage, F. H. (2005). Exercise enhances learning and hippocampal neurogenesis in aged mice. J. Neurosci. 25, 8680-8685. doi: 10.1523/JNEUROSCI.1731-05.2005

Rancan, M., Morganti-Kossmann, M. C., Barnum, S. R., Saft, S., Schmidt, O. I., Ertel, W., et al. (2003). Central nervous system-targeted complement inhibition mediates neuroprotection after closed head injury in transgenic mice. J. Cereb. Blood Flow Metab. 23, 1070-1074. doi: 10.1097/01.WCB.0000084250.20 $114.2 \mathrm{C}$
Reichwald, J., Danner, S., Wiederhold, K.-H., and Staufenbiel, M. (2009). Expression of complement system components during aging and amyloid deposition in APP transgenic mice. J. Neuroinflammation 6:35. doi: 10.1186/17422094-6-35

Shen, Y., Li, R., McGeer, E. G., and McGeer, P. L. (1997). Neuronal expression of mRNAs for complement proteins of the classical pathway in Alzheimer brain. Brain Res. 769, 391-395. doi: 10.1016/S0006-8993(97)00850-0

Smyth, G. K. (2004). Linear models and empirical bayes methods for assessing differential expression in microarray experiments. Stat. Appl. Genet. Mol. Biol. 3, Article3. doi: 10.2202/1544-6115.1027

Smyth, G. K., Michaud, J., and Scott, H. S. (2005). Use of within-array replicate spots for assessing differential expression in microarray experiments. Bioinformatics 21, 2067-2075. doi: 10.1093/bioinformatics/bti270

Sousa-Victor, P., Gutarra, S., Garcia-Prat, L., Rodriguez-Ubreva, J., Ortet, L., RuizBonilla, V., et al. (2014). Geriatric muscle stem cells switch reversible quiescence into senescence. Nature 506, 316-321. doi: 10.1038/nature13013

Stephan, A. H., Madison, D. V., Mateos, J. M., Fraser, D. A., Lovelett, E. A., Coutellier, L., et al. (2013). A dramatic increase of Clq protein in the CNS during normal aging. J. Neurosci. 33, 13460-13474. doi: 10.1523/JNEUROSCI.1333-13.2013

Stilling, R. M., Ronicke, R., Benito, E., Urbanke, H., Capece, V., Burkhardt, S., et al. (2014). K-Lysine acetyltransferase 2a regulates a hippocampal gene expression network linked to memory formation. EMBO J. 33, 1912-1927. doi: 10.15252/embj.201487870

Subramanian, A., Tamayo, P., Mootha, V. K., Mukherjee, S., Ebert, B. L., Gillette, M. A., et al. (2005). Gene set enrichment analysis: a knowledge-based approach for interpreting genome-wide expression profiles. Proc. Natl. Acad. Sci. U.S.A. 102, 15545-15550. doi: 10.1073/pnas.05065 80102

Takahashi, H., McCaffery, J., Irizarry, R., and Boeke, J. (2006). Nucleocytosolic acetyl-coenzyme a synthetase is required for histone acetylation and global transcription. Mol. Cell 23, 207-217. doi: 10.1016/j.molcel.2006.05.040

Terai, K., Walker, D. G., McGeer, E. G., and McGeer, P. L. (1997). Neurons express proteins of the classical complement pathway in Alzheimer disease. Brain Res. 769, 385-390. doi: 10.1016/S0006-8993(97)0 0849-4

Tollervey, J. R., Wang, Z., Hortobágyi, T., Witten, J. T., Zarnack, K., Kayikci, M., et al. (2011). Analysis of alternative splicing associated with aging and neurodegeneration in the human brain. Genome Res. 21, 1572-1582. doi: $10.1101 /$ gr. 122226.111

Twine, N. A., Janitz, K., Wilkins, M. R., and Janitz, M. (2011). Whole transcriptome sequencing reveals gene expression and splicing differences in brain regions affected by Alzheimer's disease. PLoS ONE 6:e16266. doi: 10.1371/journal.pone.0016266

van Praag, H., Christie, B. R., Sejnowski, T. J., and Gage, F. H. (1999). Running enhances neurogenesis, learning, and long-term potentiation in mice. Proc. Natl. Acad. Sci. U.S.A. 96, 13427-13431. doi: 10.1073/pnas.96.23. 13427

Verbitsky, M., Yonan, A. L., Malleret, G., Kandel, E. R., Gilliam, T. C., and Pavlidis, P. (2004). Altered hippocampal transcript profile accompanies an age-related spatial memory deficit in mice. Learn. Mem. 11, 253-260. doi: 10.1101/lm. 68204

Verdaguer, E., Junyent, F., Folch, J., Beas-Zarate, C., Auladell, C., Pallas, M., et al. (2012). Aging biology: a new frontier for drug discovery. Expert Opin. Drug Discov. 7, 217-229. doi: 10.1517/17460441.2012.660144

Wood, S. H., Craig, T., Li, Y., Merry, B., and de Magalhaes, J. P. (2013). Whole transcriptome sequencing of the aging rat brain reveals dynamic RNA changes in the dark matter of the genome. Age (Dordr) 35, 763-776. doi: 10.1007/s11357012-9410-1

Wyss-Coray, T., Yan, F., Lin, A. H.-T., Lambris, J. D., Alexander, J. J., Quigg, R. J., et al. (2002). Prominent neurodegeneration and increased plaque formation in complement-inhibited Alzheimer's mice. Proc. Natl. Acad. Sci. U.S.A. 99, 10837-10842. doi: 10.1073/pnas. 162350199

Xu, X., Zhan, M., Duan, W., Prabhu, V., Brenneman, R., Wood, W., et al. (2007). Gene expression atlas of the mouse central nervous system: impact and interactions of age, energy intake and gender. Genome Biol. 8, R234. doi: 10.1186/gb-2007-8-11-r234

Zahn, J. M., Poosala, S., Owen, A. B., Ingram, D. K., Lustig, A., Carter, A., et al. (2007). AGEMAP: a gene expression database for 
aging in mice. PLoS Genet. 3:e201. doi: 10.1371/journal.pgen.00 30201

Zambelli, F., Pesole, G., and Pavesi, G. (2009). Pscan: finding over-represented transcription factor binding site motifs in sequences from co-regulated or coexpressed genes. Nucleic Acids Res. 37, W247-W252. doi: 10.1093/nar/gkp464

Conflict of Interest Statement: The authors declare that the research was conducted in the absence of any commercial or financial relationships that could be construed as a potential conflict of interest.

Received: 27 August 2014; accepted: 21 October 2014; published online: 13 November 2014.
Citation: Stilling RM, Benito E, Gertig M, Barth J, Capece V, Burkhardt S, Bonn S and Fischer A (2014) De-regulation of gene expression and alternative splicing affects distinct cellular pathways in the aging hippocampus. Front. Cell. Neurosci. 8:373. doi: 10.3389/fncel.2014.00373

This article was submitted to the journal Frontiers in Cellular Neuroscience. Copyright (ㅇ 2014 Stilling, Benito, Gertig, Barth, Capece, Burkhardt, Bonn and Fischer. This is an open-access article distributed under the terms of the Creative Commons Attribution License (CC BY). The use, distribution or reproduction in other forums is permitted, provided the original author(s) or licensor are credited and that the original publication in this journal is cited, in accordance with accepted academic practice. No use, distribution or reproduction is permitted which does not comply with these terms. 